\title{
DIFFRACTION AND HOLOGRAPHY WITH PHOTOELECTRONS AND AUGER ELECTRONS: SOME NEW DIRECTIONS
}

Charles S. Fadley

\author{
University of California-Davis \\ Davis, C.A 95616 \\ and \\ Materials Sciences Division \\ Lawrence Berkeley Laboratory \\ University of California \\ Berkeley, California 94720
}

June 1992

This work was supported by the Office of Naval Research under Contract N00014-90-J-1457 and by the Director, Office of Energy Research, Office of Basic Energy Sciences, Materials Sciences Division, of the U.S. Department of Energy under Contract No. DE-AC03-76SF00098. 
To appear in Surface science Reports

Proceedings of the Tenth

International summer Institute in surface science

Milwaukee, Wisconsin, 29 June-2 JulY, 1992

\title{
DIFFRACTION AND HOLOGRAPHY WITH PHOTOELECTRONS AND AUGER ELECTRONS: SOME NEW DIRECTIONS
}

\author{
Charles S. Fadley \\ University of California-Davis \\ Davis, CA 95616 USA \\ and \\ Lawrence Berkeley Laboratory \\ Materials Science Division \\ Berkeley, CA 94720 USA
}

\begin{abstract}
The current status of photoelectron and Auger-electron diffraction is reviewed, with emphasis on new directions of activity. The use of forward scattering in the study of adsorbed molecules, epitaxial overlayers, and clean surfaces is one of the most developed applications, and one that will become more powerful as higher energy resolution and perhaps spin analysis are used to resolve emitters on the basis of chemical state, position at a surface, or magnetic state. The use of larger data sets spanning a considerable fraction of the solid angle above a surface will also much enhance the structural information available, for example, in the growth of epitaxial layers or nanostructures on surfaces. Detailed fitting of experimental data to theoretical calculations based upon either single scattering or multiple scattering should also provide more rich structural information, including such parameters as substrate interlayer relaxation. Surface phase transitions in which near-surface layers become highly disordered can also be studied, with results that are complementary to those
\end{abstract}


from such techniques as low energy electron diffraction and medium energy ion scattering. Short-range magnetic order also can be probed by somehow resolving the spin of the outgoing electrons, e.g. by using multiplet-split core levels. Valence levels also are found to exhibit core-like diffraction effects in cases for which there is somehow rather complete integration over the bands involved, e.g., through working at higher photon energies, higher temperatures and/or integrating over energy in spectra. The possibility of holographically analyzing large-scale diffraction data sets so as to directly yield three-dimensional atomic images is also promising for certain types of problems, especially adsorbates or thin overlayers. Although several types of aberrations and artifacts arise with such holographic images, a number of correction procedures appear possible, and tests of these in model calculations and for a few sets of experimental data are encouraging. Although the application $c$ this type of analysis to multilayer substrate emission is still somewhat problematic in showing atomic images that are severely elongated, this is not necessarily true for adsorbate emission. A recent experimental and theoretical study of an adsorbate using a selected data range yields promising results. Finally, theoretical calculations indicate that it should also be possible to apply the holographic methodology to the direct imaging of short-range magnetic order. 


\section{INTRODUCTION:}

Photoelectron diffraction (PD) and its close relative Auger electron diffraction (AED) have by now been developed as quantitative surface structure probes to the extent that an ever-increasing number of groups is making use of them. Several comprehensive reviews have appeared [1-5], and these together present an up-to-date picture of the field as of about 1-2 years ago. The aim of this paper will thus be to briefly introduce these techniques and what has already been learned about and with them, and then to consider in more detail several recent developments and possible future directions, including the relatively newly suggested holographic analysis of such diffraction data $[6,7]$. Stress will also be placed on pointing out certain unique features of such measurements as judged against the several other surface structure techniques that are currently in use. A more extensive discussion of general experimental aspects and theoretical background, as well as a more complete bibliography, appear in a detailed overview by the author that has very recently been published [5].

The basic measurement involved is very simple and is illustrated in Fig. 1. In PD, a photoelectron is emitted from a core level and in AED, an Auger electron is emitted in a process involving at least one core level. Thus, the energy of the outgoixig electron is unique to a given atomic type (i.e., atomic number), or, witbl high enough energy resilution, also perhaps to a given chemical or magnetic state of a given atomile type (as will be discussed in more detail below). The intensity of thi.s photoelectron or Auger electron is then measured as a function of direction above a singlecrystal surface, or, for photoelectrons, also perhaps as a function of the photon energy used for excitation. The former we shall refer to as a "scanned-angle" measurement, and the latter as "scanned-energy". The observed variations in intensity with either direction or energy are due to scattering of the outgoing "direct" wave $\phi_{O}$ from various atoms surrounding the emitter; the interference of this direct wave and various singly- and 
multiply-scattered waves $\phi_{j}$ then produces a diffraction pattern. This diffraction pattern can then be analyzed at several levels so as to deduce structural information concerning the near-neighbor atoms around a given type of emitter, as well as the atomic composition [1-9].

A number of prior studies have lead to several general points that can be made concerning such diffraction patterns:

-The variations in intensity can be very large, reaching 50-70\% as measured by the normalized anisotropy $\left[I_{\operatorname{maximum}}-I_{\operatorname{minimum}}\right] / I_{\operatorname{maximum}} \equiv$ $\Delta I / I_{\max }[5]$. Thus, these diffraction patterns can be relatively easily measured. They also cannot be neglected in any attempt to determine surface compositions or sioichiometries from single-crystal $x$-ray photoelectron (XPS) intensities, as errors of more than a factor of 2 can result [8].

-Especially at higher kinetic energies of $1 \mathrm{keV}$ or more, the diffraction features can be as narrow as a few degrees in full width at half maximum intensity (FWEM) [5]. Thus, measurements at higher angular resolutions of $\simeq \pm 1.0^{\circ}$ may be necessary to derive all of the structural information possible, although many studies to date have also been fruitfully carried out at lower resolutions of $\approx \pm 3.0-6.0^{\circ}$.

-The direct wave $\phi_{0}$ in a photoelectron measurement is more easily defined in a quantitative sense, as it is caused by a dipole excitation from some initial angular momentum 1 to the two allowed and interfering finalstate channels of $1 \pm 1$. The exact mixing of these $1 \pm 1$ components can be calculated, provided that the relevant radial matrix elements and phase shifts are available $[5,9(a), 9(c), 9(d), 10]$. By contrast, the coulomb and exchange interactions responsible for Auger decay can lead to non-zero contributions from various final-state channels, although in some cases, it can be argued that one channel may dominate [11]. As a limiting, but not necessarily accurate, approximation for the direct wave in Auger emission, it has sometimes also been assumed that the mixing of many 1 components 
leads to an effectively s-wave character $[5,9(c), 11-13]$, and we will discuss some model theoretical calculations based on this assumption below.

-The more complex, often multipeak, nature of Auger spectra in general makes the use of higher energy resolution to resolve chemical and/or magnetic states easier in photoelectron spectra. This fact, plus the better defined nature of the outgoing direct wave, will lead us here to focus more on photoelectron diffraction and holography, even though much of what is useful in these techniques can also be said of Auger electron diffraction and holography.

-The electron-atom scattering which produces the diffraction pattern is dominated at higher energies of $1 \mathrm{kev}$ or more by a strong and narrow peak in the forward direction, while at lower energies of approximately 50-300 eV it can be strong for all angles from forward- to back-scattering [5]. Thus, measurements in the typical XPS regime often take advantage of strong forward scattering peaks to derive bond- or low-index-directions relative to a given emitter type, whereas those at lower energies (and usually involving synchrotron radiation for excitation) can make use of back-scattering or side-scattering events to derive information on atoms "behind" or "beside" the emitter as viewed from the detector.

-Photoelectron diffraction appears to be ratber accurately described for many cases by a single-scattering (kinematical) model, even though multiple-scattering (dyramical) effects can be quite important for certain geometries and certain energies. For example, marked intensity reductions can occur for emission along chains of atoms due to repeated forward scattering at higher energies [9(c),12,13], as first pointed out by Tong and co-workers [12]; this has been termed multiple scattering "defocussing" from its analogue in classical optics. single scattering calculations that do not include these defocussing effects are thus often found to predict too high intensities along low-index directions above surfaces, even though the remainder of the diffraction features away from these directions may be 
rather accurately described. As an example of this, we show in Fig. 2 a nearly full hemisphere of Si 2p XPS intensities above a si(111) surface, together with a single-scattering calculation of the same diffraction pattern [14]. The two patterns have been normalized to have the same average heights over the regions away from the strong forward scattering peaks. The peaks along the lowest-index directions labelled are too strong and too broad in the calculation, but an inspection of the remaining fine structure shows that it is very well predicted by single scattering. Similar conclusions have been reached by osterwalder and co-workers based on the measurement of a number of full-hemisphere diffraction patterns above metal surfaces and epitaxial overlayers [15].

-The single scattering photoelectron or Auger electron intensity as a function of wave vector can be written as:

$$
\begin{aligned}
I(\underline{k}) & \propto\left|\phi_{o}+\Sigma_{j} \phi_{j}\right|^{2} \\
& \propto\left|\phi_{o}\right|^{2}+\Sigma_{j}\left(\phi_{o}{ }^{*} \phi_{j}+\phi_{o} \phi_{j}{ }^{*}\right)+\Sigma_{j} \Sigma_{k} \phi_{j} \phi_{k}{ }^{*},
\end{aligned}
$$

where $k$ is the electron wave vector and $\phi_{j}$ and $\phi_{k}$ are arbitrary scattered waves. For the simple reference case of photoelectron emission from an $s$ subshell into an outgoing $\phi_{O}$ with $p$ character, the individual wave components here can be written out more explicitly in terms of (cf. Fig. 1): dipole matrix elements proportional to the radiation polarization direction (k) dotted into the relevant emission direction $\left(\underline{k} / k=\mathbb{k}\right.$ or $\underline{\underline{r}}_{j} / x_{j}=$ $\left.\hat{r}_{j}\right) ;$ exponential decay factors $\exp \left(-I_{s} / 2 \Lambda_{e}\right)$, with $L$ equal to the total length for some path below the surface and $\Lambda_{e}$ the inelastic attenuation length; scattering factors $f_{j}\left(\theta_{j}\right)$ involving both an amplitude $\left|f_{j}\left(\theta_{j}\right)\right|$ and a phase shift $\Psi_{j}\left(\theta_{j}\right)$ that are functions of the scattering angle $\theta_{j}$; Debyewaller factors $w_{j}$ that allow for attenuation of interference due to vibrational effects; and finally, the phase shifts due to path length differences of the form $\exp \left[i k r_{j}\right] \exp \left[-i \underline{k} \cdot \underline{r}_{j}\right]=\exp \left[i k r_{j}\left(1-\cos \theta_{j}\right)\right]$. All 
structural information is thus contained in these last factors, with the path length difference between $\phi_{0}$ and $\phi_{j}$ being given by $r_{j}\left(1-\cos \theta_{j}\right)$. Eq. 1 then becomes:

$$
\begin{gathered}
I(\underline{k}) \propto\left|(\hat{\xi} \cdot \bar{k}) \exp \left(-L_{o} / 2 \Lambda_{e}\right)+\Sigma_{j}\left(\hat{\xi} \cdot \hat{r}_{j} / x_{j}\right)\right| f_{j}\left(\theta_{j}\right) \mid w_{j} \exp \left(-L_{j} / 2 \Lambda_{e}\right) \\
\quad \times\left.\exp \left[i\left\{k x_{j}\left(1-\cos \theta_{j}\right)+\Psi_{j}\left(\theta_{j}\right)\right\}\right]\right|^{2},
\end{gathered}
$$

or, in more convenient notation:

$$
I(\underline{k}) \propto\left|F_{0}+\Sigma_{j} F_{j} \exp \left[-i \underline{k} \cdot \underline{r}_{j}\right]\right|^{2},
$$

with

$$
\begin{aligned}
& F_{0}=(\hat{\epsilon} \cdot k) \exp \left(-L_{0} / 2 \Lambda_{e}\right) \\
& F_{j}=\left(\varepsilon \cdot \hat{x}_{j} / r_{j}\right)\left|f_{j}\left(\theta_{j}\right)\right| w_{j} \exp \left(-L_{j} / 2 \Lambda_{e}\right) \exp \left[i \Psi_{j}\left(\theta_{j}\right)\right] \exp \left[i k r_{j}\right] .
\end{aligned}
$$

Here, one portion of the phase factor due to path length (exp[ikx $j]$ ) is now incorporated into the $F_{j}$ 's. Eq. 3 can also be formally generalized to include multiple scattering $[7]$, in which case we must include in each $F_{j} a$ sum over the various single and multiple scattering pathways m with different total lengths $I_{m j}$ that terminate in scatterer $j$ just before going to the detector; within each multiple-scattering pathway, there also will be products of successive path-length phase factors and scattering factors. Expanding the square in Eq. 3 then yields

$$
\begin{aligned}
I(\underline{k}) & \propto\left|F_{0}\right|^{2}+\Sigma_{j}\left[F_{0}{ }^{*} F_{j} \exp \left\{-i \underline{k} \cdot \underline{r}_{j}\right\}+F_{o} F_{j}{ }^{*} \exp \left\{i \underline{k} \cdot \underline{\underline{r}}_{j}\right\}\right] \\
& +\Sigma_{j} \Sigma_{k}\left[F_{j}{ }^{*} F_{k} \exp \left\{i \underline{k} \cdot\left(\underline{r}_{j}-\underline{\underline{r}}_{k}\right)\right\}+F_{j} F_{k}{ }^{*} \exp \left\{-i \underline{k} \cdot\left(\underline{r}_{j}-\underline{\underline{r}}_{k}\right)\right\}\right\} .
\end{aligned}
$$

$\left|F_{0}\right|^{2} \equiv I_{0}$ is just the intensity in the absence of any scattering. This form will be useful when we later consider holographic analyses of 
diffraction. One common approximation is to assume that the scattered waves $\phi_{j}$ and $\phi_{k}$ are small in amplitude with respect to $\phi_{o^{\prime}}$ so that the cross terms $\phi_{0}{ }^{*} \phi_{j}$ and $\phi_{0} \phi_{j}{ }^{*}$ in Eq. 1 contain all of the structural information. In Eq. 4, this implies neglecting the double sum over $j$ and $k$, and then straightforwardly leads with Eqs. 2 and 3 to:

$$
\begin{gathered}
I(\underline{k}) \propto\left|F_{o}\right|^{2}+2 F_{o} \Sigma_{j}\left(\varepsilon \cdot \hat{r}_{j} / r_{j}\right)\left|f_{j}\left(\theta_{j}\right)\right| w_{j} \exp \left(-I_{j} / \Lambda_{e}\right) \\
x \cos \left[k r_{j}\left(1-\cos \theta_{j}\right)+\Psi_{j}\left(\theta_{j}\right)\right] .
\end{gathered}
$$

This form is relevant when considering how Fourier transforms of scannedenergy data can be used to derive path length differences $[9(b), 16]$. Finally, we note that the presence of the surface potential barrier or inner potential $v_{0}$ can lead to electron refraction of both the direct and scattered waves as they leave the surface, and that the detection system will in general integrate over some range of emission directions as set by its acceptance solid angle of $\Omega_{0}$ (cf. Fig. 1).

-single-scattering is also probably a good approximation for much of Auger electron diffraction, but it is more difficult to assess theoretical models for this case due to the more complex nature of the outgoing direct wave, which may consist of a complex mixture of orbital angular momenta. For the simplifying assumption of an s outgoing wave in Auger emission, Eq. 2 can be modified simply by eliminating the dot-product factors due to dipole matrix elements.

-Full multiple scattering calculations are being carried out routinely for both PD and AED results $[9,11-13]$, and, with the exception of adequately defining the outgoing wave in Auger emission, these are no more complicated in principle than the calculations required for analyzing low energy electron diffraction (LEED) data. However, one expects a single-scattering picture to be more quantitatively valid for many cases in $P D$ and AED than it is in general in IEED. 
-The most appropriate theoretical models for simulating either PD or AED data are based on a short-range-order cluster approach, in which single or multiple scattering is computed only over some set of near-neighbor atoms to a given emitter [Fig. 1 and refs, 1,2,5,9(b)-9(d)]. Depending on the energy and the geometry of the problem, the number of atoms that need to be included in such a cluster can range from only a few to as many as a few hundred, with the final criterion on cluster size being that the diffraction pattern should be stable as size is further increased. It is here also important to stress that both PD and AED are principally probes of shortrange-order, with the first couple of spheres of neighbors usually producing most of the strongest features in the diffraction patterns, and spheres out to 15-20 a ay contributing only to additional fine structure on these features. This cluster approach can be contrasted to the long-range-order methods applied traditionally in LEED analyses [9(a),17]. In a typical IEED measurement, it is order over regions of about $100 \AA$ in diameter that controls spot patterns, making such methods a natural choice for theoretical calculations. However, it has recently been shown experimentally by Heinz and co-workers [18] that, even in LEED, the energy variation of spot intensities is controlled by short-range order. An advantage of the longrange order models in treating either IEED or photoelectron diffraction is in being able to more easily deal with multilayer substrate scattering by layer-doubling schemes [17]; in the clustex approach, substrate emission must often be modelled by using extremely large clusters.

\subsection{SOME CURRENT AND FUTURE APPLICATIONS:}

We here consider some more or less well-established areas of application of $P D$ and $A E D$ to illustrate the types of information that can be derived, commenting on how these are likely to be extended in the future.

\subsection{Forward scattering in adsorbed molecules:}


In adsorbed molecules, if one atom lies below another as viewed from the detector, then the latter can act as a forward scatterer at higher energies and thus produce a strong peak in diffraction patterns exactly along the internuclear axis. This was first demonstrated for verticallyoriented co on $\mathrm{Ni}(001)$ [19], and such effects have subsequently been used with considerable success on several other adsorbate systems, especially by Bonzel and co-workers [20].

As one example of this type of study, we show in Fig. 3 polar and azimuthal scanned-angle dats for $\mathrm{C}$ 1s emission from the $\alpha_{3}$ state of co on Fe(001) that have been obtained by Saiki et al. [21]. The polar-angle data of Fig. 3(a) have been normalized by dividing with the 0 ls intensity from the same molecule in order to eliminate purely instrumental variations with angle. In these polar data, the strong forward scattering peak at $55^{\circ}$ from the surface normal $\left(35^{\circ}\right.$ from the surface plane) immediately suggests that the co molecule is tilted at this angle as well. setting the polar orientation at this tilt and scanning in azimuthal angle then leads to the results in Fig. $3(\mathrm{~b})$, which further indicate that the co molecules have preferred orientations along the <100> azimuths, probably ix four equallypopulated domains. The final structural model for the local bonding of each co on this surface is thus as shown in Fig. 3(c). We note that the direct nature of the interpretation of these forward scattering peaks permits making these structural conclusions without resort to any sort of theoretical modeling, and is a distinct advantage of this approach. Nonetheless, calculations at both the single-scattering and multiplescattering levels for the geometry of Fig. $3(c)$ are found to agree very well with experiment [21].

As a second example that has important implications for future applications with higher energy resolution, we show in Fig. $4 N$ is core spectra from $\mathrm{N}_{2}$ on $\mathrm{Ni}(001)$ due to Nilsson, Tilborg, and Mårtensson [22]. Here the two $N$ atoms exhibit a chemical shift, with the nitrogen atom $N$ bound directly to the $\mathrm{Ni}$ substrate in an atop geometry having a binding 
energy that is about $1.3 \mathrm{eV}$ greater than that of the second atom $N^{\prime}$ facing outward from the surface. Measuring the intensity ratio of these two peaks as a function of polar angle (in just the same way as the cls/ols ratio was used in Fig. 3(a)) then shows very directly in the inset that this molecule is oriented normal to the surface, and by implication also identifies the higher-binding-energy peak as being associated with the lower $N$ atom that can exhibit forward scattering. This use of chemical shifts to study independently the local structure around inequivalent atoms of a given type is a major advantage of photoelectron diffraction that should become more widely used, particularly in conjunction with high-resolution synchrotron radiation sov : ses. We will return to this topic below.

\subsection{Forward scattering and diffraction from epitaxial overlayers and clean surfaces:}

Higher-energy PD and AED measurements have by now been made for numerous cases of epitaxial growth on single-crystal substrates, and this type of work has been reviewed by Chambers et al. [3], Egelhoff [4], and the author $[2,5]$. In such studies, the forward scattering peaks along nearneighbor directions that are also often incipient low-index directions in an epitaxial overlayer can be used to determine not only how many layers of growth one has, but also to decide from the polar angle at which these peaks are observed whether there have been vertical relaxation in the interlayer distances during growth. But it is also desirable to go further in the analysis of such data and compare experimental diffraction patterns with theoretical predictions for the assumed geometry, as the more complex diffraction features away from the strongest forward scattering directions also contain useful structural information.

As a first example of thi.s kind of analysis, we consider results due to Herman et al. for an epitaxially-grown $\mathrm{Hg}_{0.78} \mathrm{Cd}_{0.22} 2^{\text {Te }}$ sample with (111) orientation [23]. In Fig. 5(a) are presented azimuthal data for core peaks from all three of the atoms present in the sample, taken at a polar angle of 
$19^{\circ}$ with respect to the surface that is chosen to pass through nearestneighbor $\langle 11,-1\rangle$ forward scattering directions. These nearest-neighbor peaks occur at $\phi=0^{\circ}$ and $120^{\circ}$ in the figure, and have the same origin as the peak for $s i$ labelled $[11,-1\}$ in Fig. $2(a)$. The experimental data are compared with single scattering calculations for two different types of termination of the surface: " $\mathbf{A}$ " = cation-terminated with $\mathrm{Cd}$ or Bg at the stifface, and "B" = anion-terminated with Te at the surface. The forward scattering peaks at $\phi=0^{\circ}$ and $120^{\circ}$ are strong in all experimental and theoretical curves; thus, no decision on the ternination could be made from these peaks alone. However, considering the additional fine structure between these peaks for both the $\mathrm{Bg}^{4 f_{7 / 2}}$ and $\mathrm{Cd} 3 \mathrm{~d}_{5 / 2}$ intensities, it is clear fron both visual and R-factor comparisons that the "A" termination is strongly preferred. For the Te $3 d_{5 / 2}$ intensity, the conparison in Fig. 5 (a) is not as conclusive, with equal R-factors for the two terminations. Bowever, if we again note that the forward scyttering peaks are expected to be overestimated in single-scattering theory, we can consider only the $\phi$ range between these two peaks, and rescale the curves to permit a more quantitative conparison over this middle range. This comparison is shown in Fig. $5(b)$, for which it is now clear that the "A" ternination better describes experiment for all thrse of the constituent atons. This work thus illustrates the power of such measurements, in combination with a relatively sinple theory, for deternining even rather subtle aspects of the surface norphology of epitaxial overlayers.

The results in Fig. 5 also illustrate a typical discrepancy seen between experimgntal data and single-scattering, or even multiple scattering, theory: the percent effect as judged by the anisotropy $\Delta I / I_{\max }$ is often predicted to be too high by as much as a factor of 3-5 [5]. Sone of the reasons for this are: residual surface disorder or other types of non-ideal enitter sites at defects or steps; lack of adequate consideration of quasi-elastic scattering by electrons or phonons that any reduce the fraction of coherent enission in a given spectral peak; and multiple scattering, particularly along low-index directions. such discreparcies are also often found in aultiple scattering analyses of LEED data [17]. 
Being able to measure these diffraction patterns separately for all of the elements present in the specimen is clearly a ke advantage for such epitaxy studies. At higher energies, the diffraction pattern is essentially a fingerprint of the type of site occupied by a given atom, and thus it should be rather straightforward to determine whether it occupies a normal lattice site, an interstitial site, or some sort of special or disordered site. In multicomponent systems or epitaxial sy lams that may show interdiffusion, this ability is also important, as an atom that, for example, preferentially diffuses to the surface, will show little or no forward scattering and a higher relative intensity than if it werc uniformly distributed through a given epilayer. By contrast, diffusion of an epitaxial component into the substrate can be very easily detected by the turning on of forward scattering effects. An example of this is o on Ni(001), for which Saiki et al. [24] wave found oxygen burial and/or oxide nucleus formation well before the completion of the $c(2 \times 2) 0$ adsorbate overlayer that is evident in the LEED spot pattern. A more recent case studied by Fischer et al. [15(b)] is that of Mg evaporated onto Pd(111). Some of the data from this work is shown in Fig. 6. Here, the fullhemisphere diffraction patterns for Mg 1 s emission at $437 \mathrm{eV}$ clearly show forward scattering peaks by a coverage of only $0.5 \mathrm{~kL}$, and they furthermore go from being threefold symmetric to being sixfold symetric between 0.9 and 1.5 $\mathrm{M}$. These data have been interpreted as implying that Mg initially occupies fcc sites distributed over the first two Pd layers, and then for higher coverages forms small oriented clusters in alternate fcc and hcp sites. Iong-range order is not present in any of these $\mathrm{Mg} / \mathrm{Pd}$ structures, so IEED is of limited value in studying this and other similar problems.

As a final comment on surface structures and epitaxy, we note that the use of chemical shifts pronises here also to permit doing site-specific diffraction measurenents in a way that is not possible with any other structural probe of which we are aware. For example, sebilleau and coworkers have first measured the separate diffraction patterns of the surface 
and bulk layers of a tungsten surface, using the binding energy shifts between them to separate the core intensities [25]. For certain cases, this thould also be possible in compound epitaxial growth as well. For example, Bimpsel et al. [26(a)] have demonstrated that it is possible with synchrotron radiation to resolve in thin oxide epilayers on $\mathrm{Si}(100)$ or Si(111) all of the oxidation states from element (0) to $\mathrm{SiO}_{2}(+4)$. This suggests the exciting future possibility of doing state-specific diffraction on il of the four oxide-related atomic types, or similar state-specific studies on other overlayers and interfaces. In fact, Denliger et al. [26(b)] have recently resolved the bulk and interface Ca $2 p$ levels in epitaxial $\mathrm{CaF}_{2}$ grown on $\mathrm{Si}(111)$, and succeeded in carrying out separate $\mathrm{XPD}$ measurements on these two components.

\section{MORE DETAILED STRUCTURAL ANALYSES AND NOVEL APPLICATIONS:}

\subsection{Multiparameter fitting of experiment and theory:}

With a sufficiently accurate and extensive photoelectron or Auger electron diffraction data set, it is possible to proceed further with a structural study so as to determine not only adsorbate site symmetries and vertical positions, but also the various substrate interlayer relaxations that are by now known to occur in response to adsorbate bonding. This would generally involve comparing experiment and theory (at either singlescattering or nultiple-scattering level) for a number of different structures, perhaps using R-factors as a quantitative indicator of goodness of fit. This procedure is thus identical in spirit to that used in all LEED structure determinations [17]. Such analyses are to date relatively few in number, and we will here give two illustrations of them as an indicator of what wight be expected in future work, one based on scanned-energy data emphasizing back scattering due to Shirley and co-workers [27], and one on higher-energy scanned-angle data due to Saiki and co-workers [28]. 
In a scanned-energy study of $(\sqrt{3} \times \sqrt{3}) R 30^{\circ} \mathrm{Cl}$ on $\mathrm{Ni}(111)$, Wang et al. [27] have been able to determine the particular threefold-hollow site in which the adsorbate sits (two distinct ones are possible), the vertical distance of the $C l$ above the surface (1.84A), and the degree of contraction of the vertical distance between the first and second Ni layers of the substrate ( $5 \%$ relative to bulk $\mathrm{Ni}$ ). As an indicator of the high sensitivity of such data to the substrate relaxation, Fig. 7 compares experiment and multiple scattering theory with (panel (a)) and without (panel (b)) any such interlayer contraction. The fit of theory to experiment is excellent with contraction, and is severely degraded without contraction. other adsorbate systems have been studied in similar detail by the shirley group using the scanned-energy approach [9(b),29], and in these also, strong sensitivity to substrate relaxation has been demonstrated.

In a scanned-angle investigation of the well-defined test case of $c(2 \times 2) s$ on $N i(001)$, Saiki et al. [28] have been able to determine both the vertical distance of $S$ above the surface (1.39 ) and the degree of expansion of the distance between the first and second Ni layers (68 relative to bulk Ni). Some R-factor curves used in arriving at the final structure are shown in Fig. 8(a). Fig. 8(b) shows the final comparison of experiment and single-scattering theory for the optimum structure, and the agreement is very good for the three highest polar angles above the surface $\left(\theta=10^{\circ}\right.$, $12^{\circ}$, and $16^{\circ}$ ). The lowest angle shows more disagreenent, and this is not surprising, as multiple forward scattering parallel to rows of $s$ atoms along the surface is expected to be more important for lower angles that lie within the forward scattering cone. This work was done at a higher energy of approximately $1085 \mathrm{ev}$ at which forward scattering is expected to be dominant, and thus the contributions of the $\mathrm{Ni}$ atoms lying below $\mathbf{s}$ are expected to be rather weak. It is thus encouraging that this wuch sensitirity is seen to not only the vertical adsorbate distance but also the substrate relaxation. Future data with better statistical accuracy and over more azimuths going to higher takeoff angles over which a simpler singlescattering picture is expected to apply should improve such structural determinations. 
A final note of caution concerning such R-factor comparisons of experiment and theory is in order however. It has been found by Saiki et al. in an XPD study of $0 / \mathrm{Ni}(001)$ [24(b)] that R-factors can exhibit oscillations as a function of certain structural parameters such as the adsorbate vertical height. Such oscillations thus enhance the probability of accidentally finding local minimum in $R$ that is not the absolute minimum, and dictate that such parameter searches be very carefully done over the full range of reasonable geometries and distances to avoid converging on an erroneous structure. (Similar oscillations in R-factors are also well known in LEED analyses of surface structures [17(c)].) The possible existence of such local minima also makes it clear that having an appioximate idea as to the geometry and atomic positions which is accurate to within even 0.5 could save a great deal of time in such structural searches, and assist considerably in avoiding such spurious structures. One

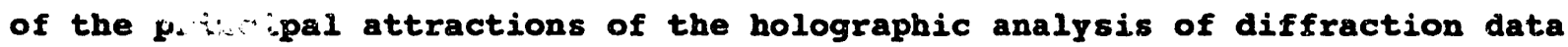
to be considered in Sec. 4 is thus that it may provide such approximate starting structures from which the final optimization can be made by fitting theory to experiment using R-factors.

\subsection{Surface-structural phase transitions:}

Next we consider the application of photoelectron diffraction to surface-structural phase transitions. Two prior studies of this type have been performed: the first on the surface melting of $\mathrm{Pb}(110)$ by Bonzel and co-workers [30] and a more recent one on a high-temperature surface phase txansition of Ge(111) by Tran et al. [31]. Both of these involved measuring the temperature dependences of certain forward scattering peaks in polar [30] or azimuthal [31] intensity scans. The study of Ge(111) also included measurements of the full intensity profile above the surface at several temperatures below and above the phase transition. We will consider the latter here as a representative example, since a more complete data set was analyzed. 
This high-temperature surface phase transition on Ge(111) was first studied using LEED by MCRae and Malic [32], who observed abrupt decreases in different spot intensities, with a saturation of the effect by about 1060K or $150 \mathrm{~K}$ below the melting point. One set of data from this study is shown in Fig. 9(a). The same transition was then monitored in photoelectron diffraction via the peak-minus-background intensity of a nearest-neighbor forward scattering peak that is found at a takeoff angle of $19^{\circ}$ with respect to the surface [31]. These data are shown in Fig. 9(b). They are similar to the LEED data in showing an abrupt drop at a temperature near 1050R, but different in that there is no saturation at this temperature, but rather a continuing drop going all the way up to the melting point. Also, the point of steepest slope is at about 1040K for LEED, and at a higher temperature of about 1060K for PD. These differences can be explained by the fact that the IEED spot intensities are primarily sensitive to long-range order on the scale of about $100 \AA$, while the $P D$ effects are sensitive to shorter-range structures on the scale of 10-20\%. Thus, the loss of long-range order would be expected to saturate at a lower temperature than that for short-range order.

The full diffraction patterns above the Ge(111) surface are shown in Fig. 10 at room temperature, just below the transition at $970 \mathrm{~K}$, and just above the transition at 1130K. With a normalization chosen so as to make ihe maximum heights of corresponding features identical, it is striking bere that all three patterns are essentially identical. This suggests that the transition leaves all emitters that are in ordered sites (and thus capable of showing diffraction) with the same near-neighbor structure. (A holographic analysis of the data in Fig. 10 using the methods to be described in sec. 4 also confirms this conclusion [31].)

Both the IEED and the PD data shown here can be explained in terms of a model in which a completely disordered liquid-like layer gradually forms on top of an underlying crystalline substrate, with the thickness of this 
layer increasing almost all of the way to the melting point, but not in the divergent manner that is found in true surface melting (as studied before for $\mathrm{Pb}(110$ ) with PD by Bonzel and co-workers [30]). Since LEED is primarily sensitive to long-range-order in the first couple of surface layers, the LEED spot intensities should drop to a very low value as soon as this disordered layer either loses its long-range coherence beyond about 30-50 or reaches a thickness of 1-2 layers. By contrast, the photoelectron diffraction features originating in the ordered substrate can be thought of as being simply attenuated in absolute intensity due to inelastic scattering in the disordered overlayex. The atoms in the disordered overlayer will to a good approximation just add a constant background intensity with no diffraction structure to any angle scan.

The PD data can then be analyzed simply by assuming an exponential attenuation according to $\exp \left[-t_{1 i q}(T) /\left(\Lambda_{e} \sin \theta\right)\right]$, where $t_{1 i q}(T)$ is the disordered overlayer thickness at a given temperature, $\Lambda_{e}$ is the inelastic attenuation length in Ge (measured for this system to be $25 \AA$ [33]), and $\theta$ is the electron takeoff angle with respect to the surface. Analyzing the intensity curve in Fig. $9(b)$ in this way yields a final "saturated" thickness of about $4 \AA$ that is in good agreement with the thickness of a single (111) double-layer of atoms of $3.3 \AA$. Also, the temperature dependence of this thickness as derived from photoelectron diffraction at two different takeoff angles of $19^{\circ}$ and $55^{\circ}$ is in excellent agreement with that found in a parallel medium energy ion scattering study of this system by Denier von der con et al. [34]. This is reasonable in view of the shortrange-order sensitivity of both techniques.

These results thus demonstrate a useful sensitivity of photoelectron diffraction (and presumably also Auger electron diffraction) to a loss of order near a surface. Although the diffraction patterns for this case (as well as for $\mathrm{Pb}(110)$ in ref. 30) have not been observed to change appreciably with temperature, this is consistent with the simple attenuation expected by a disordered overlayer. In future studies with better statistics, it would 
be interesting to look for subtle differences in these patterns with changes in temperature or direction that could provide information on the exact nature of the disordering transition. In fact, Breuer et al. [30] have speculated on this already for $\mathrm{Pb}(110)$. It may also be possible to study more subtle surface rearrangements or reconstructions, especially by going to more grazing takeoff angles to emphasize the near-surface region.

\subsection{Short-range magnetic-order near surfaces:}

The addition of spin resolution to the photoelectron diffraction or Auger electron diffraction measurement represents the final physical variable defining the outgoing electron. Spin can be defined either with an external spin detector, incurring about a $10^{4} x$ increase in data acquisition times, or with a simple internally-referenced multiplet splitting such as that illustrated in Fig. 11(a). In the latter, the L-s coupling occurring in the final state after Mn 3 s emission from $\mathrm{Mn}^{2+}$ in an ionic compound such as $\mathrm{KMnF}_{3}$ splits apart the spin-up and spin-down photoelectron peaks in energy, so that a simple measurement of the spectrum yields peaks with predominantly one or the other spin character. For this case, these photoelectron spins are furthermore referenced to the valence spin (magnetic moment) of the enitting atom. These two peaks might then be expected to exhibit different spin-dependent scattering in a magnetically-ordered environment, or more particularly to be sensitive to changes in this magnetic order with temperature, an effect that was first discussed theoretically by sinkovic and Fadley [35].

In Fig. $11(\mathrm{~b})$ are shown results due to sinkovic et al. [36(a)] in which the spin-up/spin-down intensity ratio was measured as a function of temperature along two directions above an antiferromagnetic $\mathbf{K M n}_{3}$ (110) surface. These data were taken at about 104 ov kinetic energy, as it is only at such low energies that magnetic effects, particularly exchange scattering, are expected to be large enough to measure $[35,36(b)]$. The spin-up/spin-down ratio shows a dramatic change at a temperature that is 
2.7x the Neel temperature, or well above the point at which long-range-order has disappeared. It has been suggested that this is the temperature at which short-range order in the first few spheres of neighbors finally disappears. A similar abrupt change has also been seen in the antiferromagnet Mno, for this case at about $4.7 \times$ the Neel temperature [37]. Both the Mn $3 s$ and Mn 3p spectra from Mno are also found to show similar effects, providing strong confirmation that these are genuine spin-dependent scattering phenomena [37].

Thus, although there is very little data available to date on what has been termed spin-polarized photoelectron diffraction (SPPD), this extension of the technique so as to achieve sensitivity to magnetic order already seems very promising. Various applications to magnetically-ordered overlayers and small clusters should be possible. The low kinetic energy required makes the further development of this technique depend on synchrotron radiation for excitation, and the relatively small differences in the diffraction of the two spins of about 10-158 also requires taking data of very good statistical accuracy. Higher brightness third-generation sources should thus lead to more systematic studies of this type.

\subsection{Valence photoelectron diffraction:}

Valence photoelectron diffraction may at first seem to be a contradiction in terms since we have supposed emission of a core electron in all the foregoing discussion. However, the connection is made via asking what soxt of intensity distribution in direction arises if we somehow average over all valence states in the Brillouin zone, so as to force the valence emission to look as core-like as possible. One can thus immediately identify two limiting cases in valence emission: the low-energy or ultraviolet photoemission (UPS) limit in which $\underline{k}$-conserving transitions in the zone lead to sharp peaks that have been used in many angle-resolved studies to measure band dispersions and Fermi surfaces; and the high-energy or $x$-ray photoemission (XPS) limit in which emission in a given direction 
somehow averages over the full Brillouin zone, thus effectively summing over many direct transitions. The latter limit can be reached by going to sufficiently high photon energies and temperatures such that phonon-assisted non-direct transitions dominate and/or by summing over energy for one or more of the usually broader features in a spectrum $[1,38,39]$. This XPS limit is also often referred to as a density-of-states limit, although matrix-element effects can still strongly modulate observed spectra relative to a density-of-states curve [1].

osterwalder et al. [39] first noted that, for $x$-ray photoemission from $A l(001)$, the $A 12 s$ core and energy-integrated valence-band intensities show very nearly identical diffraction patteras as a function of azimuthal angle. Aluminum is furthermore a case for which essentially full zone averaging should occur even at room temperature in XPS [38]. A somewhat more interesting case intermediate between the UPs and XPS limits is that of tungsten, as studied recently by Herman et al. [40]. For this case, $x-r a y$ photoemission from the $W 4 f$ and valence bands was studied as a function of temperature. A set of valence spectra at room temperature and at $803 \mathrm{~K}$ are shown in Fig. 12. At room temperature, the relative intensities of the two main components are found to change rapidly with azimutial angle, and this variation has been previously verified to be due to direct-transition effects and a resultant non-uniform sampling of the Brillouin zone [38]. On going to $803 \mathrm{~K}$ however, the spectral shape ceases to vary, presumably due to essentially complete zone averaging. However, if the energy-integrated absolute core and valence peak intensities are plotted against azimuth as in Fig. 13, we see strong residual diffraction effects in both curves, with the core and valence diffraction patterns being very similar, but not identical, at either temperature. Thus, it is concluded that the outgoing valence electrons scatter and diffract in essentially the same way as core electrons, but in a situation where the full zone is not averaged over, direct-transition effects can alter the spectral profile significantly. However, the short-range-order diffraction is always present in modulating whatever direct-transition peak may emerge from the surface. The residual 
differences between the $W$ if and valence azimuthal distributions even at 803K have furthermore been explained by the different angular momenta in the outgoing direct wave (core $4 f \rightarrow d+h$ as compared to predominantly valence $5 d$ $\rightarrow p+f)$.

Thus, valence photoelectron intensity distributions can under certain circumstances behave very much like core photoelectron diffraction patterns, and any analysis of their absolute intensities should allow for this. It is also interesting to note the close physical analogy between IEED and valence photoelectron diffraction in that long-range order controls the positions in k-space of the Bragg-like events (beams in IFED or direct-transition peaks in photoemission), whereas short-range order controls the $\underline{k}$ dependence of absolute peak intensities in LEED [18], diffuse LEED [18], and valence photoemission.

Finally, it has been pointed out by stuck et al. [41] that, in compounds with multiple-atom contributions to the valence spectra, it may be possible to use core diffraction patterns from the constituent atoms, together with corresponding valence diffraction patterns, to decompose the valence spectra into their different components or partial densities of states. This method has also been reviewed elsewhere [2(b)]. For this decomposition to be fully accurate, one must implicitly assume that the core and zone-averaged valence diffraction components associated with a given atom are identical; but Fig. 13 and its analysis [38] indicate that this need not be precisely true, largely due to the different angular momentum characters of the core and valence final states. Further tests of this idea for different systems are thus needed to assess its generality.

\section{HOLOGRAPHIC IMAGING FROM DIFFRACTION DATA:}

\subsection{Introduction:}

A final recent development in surface structure studies with photoelectron diffraction and Auger electron diffraction lies in the 
realization by szöke [6] that such data can be interpreted in a holographic sense, and inverted by mathematical means so as to possibly yield threedimensional images around a given emitter type. The iirst theoretical demonstration of such inversions was carried out by Barton [7], who also showed that simple three-dimensional, or even two-dimensional, Fourier-like integrals could in principle be used to generate atomic images relative to the emitter. The first atomic images to be derived from experimental data were for backscattered Rikuchi electrons and Auger emission from Cu(001) and were due to Harp, Saldin and Tonner $[42,43]$. Several exploratory studies of such holographic imaging have subsequently been performed [44-57], and we will here briefly review the principal assumptions and techniques used in them, as well as the positive and negative features of such analyses as illustrated in two specific examples.

The holographic approach begins by first noting that we can identify the direct or unscattered wave $\phi_{O}$ with the reference wave necessary for producing a hologram, and the various scattered waves $\phi_{j}$ with the subject(object) waves which interfere with the reference to produce the hologram. Since each core-associated photoelectron or Auger-electron emission process (or Kikuchi backscatiering process) is independent, the reference wave should indeed be a coherent source localized at a certain center. The hologram is then just the intensity $I(k)$, as measured over a range of solid angles, and possibly also energies.

A normalized intensity function $\chi(\underline{k})$ is now calculated, very much as in the analysis of extended $x$-ray absorption fine structure (EXAFs):

$$
x(\underline{k})=\left[I(\underline{k})-I_{0}(\underline{k})\right] / I_{0}(\underline{k})^{1 / 2}
$$

where $I_{0}(k)=\left|F_{0}\right|^{2}$ is the intensity in the absence of any scatterers, as defined previously. From Eq. 4, this yields 
(2)

$$
\chi(\underline{k}) \propto \Sigma_{j}\left[F_{0}(\underline{k})^{*} F_{j}(\underline{k}) \exp \left\{-i \underline{k} \cdot \underline{r}_{j}\right\}+F_{0}(\underline{k}) F_{j}(\underline{k}) * \exp \left\{i \underline{k} \cdot \underline{r}_{j}\right\}\right]
$$

$$
+\Sigma_{j} \Sigma_{k}\left[F_{j}(\underline{k}){ }^{*} F_{k}(\underline{k}) \exp \left\{i \underline{k} \cdot\left(\underline{r}_{j}-\underline{r}_{k}\right)\right\}+F_{j}(\underline{k}) F_{k}(\underline{k}) * \exp \left\{-i \underline{k} \cdot\left(\underline{r}_{j}-\underline{r}_{k}\right)\right\}\right] \cdot
$$

The simplest imaging procedure now makes use of the Helmholtz-Rirchoff theorem from optics to calculate the atomic image $U(\underline{r})$ (actually the source wavefield) associated with the hologram from [7]:

$$
v(x, y, z) \propto\left|\iint_{S} \chi(\underline{k}) \exp [i \underline{k} \cdot \underline{\underline{r}}] \mathrm{d} \sigma_{k}\right|,
$$

where the integral on the direction of $\underline{k}$ is over the spherical surface on which the hologram is measured. Note that we have here multiplied $\chi(\underline{k})$ by the complex conjugate of the direction-dependent part of the phase factor due to path length difference $\exp [-i \underline{k} \cdot \underline{r}]$, and that the magnitude of $\underline{k}$ is fixed. Further taking the $z$ axis to be along the symmetry axis of the hologram and thus usually also to be perpendicular to the surface and then projecting $\chi(\underline{k})$ onto the $k_{x}, k_{y}$ surface plane permits doing a two-dimensional Fourier transform with $z$ as a variable parameter to yield the image $U$ in a given 2 plane as [7]:

$$
u(x, y, z) \propto\left|\iint\left\{x(\underline{k}) \exp \left[i k_{z} z\right]\right\} \exp \left[i\left(k_{x} x+k_{y} y\right)\right] d k_{x} d k_{y}\right| .
$$

If the full opruing angle of the hologram as centered on the z-axis normal to the surface is defined to be $\alpha$, it can further be shown [7] that the uncertainties with which positions can be determined in the three coordinates are given by: $\Delta x=\Delta y=1.22 \pi /[k \sin (\alpha / 2)]=0.61 \lambda e^{/ \sin (\alpha / 2)}$ in the surface plane and $\Delta z \simeq 4 \pi /\left[k \sin ^{2}(\alpha / 2)\right]=2 \lambda_{e} / \sin ^{2}(\alpha / 2)$ perpendicular to the surface plane, where $\lambda_{e}$ is the electron de Broglie wavelength. These uncertainties can also be inversely related via the Uncertainty Principle to the ranges $\Delta k_{x^{\prime}}, \Delta k_{y}$, and $\Delta k_{z}$ that are spanned by the hologram [ $47(b)$ ]. 
The single sum in Eq. 7 over terms of types (1) and (2) represents the usual hologram of optical holography and the double sum over terms of types (3) and (4) the self-interference or self-hologram $[7,44]$. If the $F_{j}$ 's of Eq. $3(c)$ do not depend on $\underline{k}$ (implying among other things s-wave scattering), then the FT would be expected to yield peaks only at $\pm \underline{r}_{j}$ and at $\pm\left(\underline{r}_{j}-\underline{r}_{k}\right)$, that is, at \pm the atomic positions (the real and twin(conjugate) images, respectively) and at \pm all of the differences of atomic positions (the image due to self-interference and its twin(conjugate)). In the further limit that $\left|F_{0}\right| \gg\left|F_{j}\right|$ for all $j$, the double sum can be neglected and only the real image and its twin will be obtained; we explore below the degree to which this holds. However, some $\left|F_{j}\right|^{\prime} s$ are very large (e.g., in forward directions at higher energies), and the dependence of both the amplitudes and phases of the $F_{j}^{\prime} s$ on $\underline{k}$ can be very strong, including forward scattering effects, back scattering effects, scattering phase shifts, and possible multiple scattering pathways that introduce additional phase factors into the $F_{j}^{\prime} s$ of $\exp \left[i k\left|\underline{x}_{m}-\underline{x}_{n}\right|\right]$ for each $m \rightarrow n$ step in a pathway. Thus, we must immediately ask whether electron-atom scattering is strong enough to produce non-negligible self-interference effects, and whether its anisotropy and multiple character can introduce additional aberrations or artifacts.

We note first a key advantage of this kind of structural analysis that is not shared with the well-developed diffraction methods for $x$-rays, electrons, and neutrons in which an external beam is directed toward the specimen, and the diffracted intensities, often in distinct spots, are measured. In these latter experiments, the reference wave is lost into some direction that is not observed, and so a holographic analysis is not possible. This is the origin of the so-called "phase problem" in these techniques. These diffraction patterns are in fact formed only by terms of types (3) and (4) in Eq. 7, which lead in one common method of analysis to the Patterson function [45]. Thus, for most systems a trial and error approach to determixing a structure is required, whereas in principle holography with localized electron emission can directly yield threedimensional atomic images, or some reasonable approximation thereto. 
Deroud potential bolography with photoelectrons and Auger electrons, it has also been pointed out that both quasielastically backscattered rikuchi electrons [42] and low energy electron diffraction from systems mithout long-range order (diffuse Is:D) [46(a)] and with long-range order (conventional IEED) [46(b)] can be trented in this way. However, as the initial $\phi_{0}$ waves in both of these cases are inherently nore complex to describo, whall not concider these directions further here.

\subsection{Expected imaging difficulties and possible correction procedures:}

studies to date in fact show several types of inage distortions and other probleas, and hare also lead to suggestions for how to correct these aberrations. Nost, but not all, of these difficulties arise because electron waves are wore complex, either in their excitation or theix scattering, than the light waves of optical holography. By contrast, visible light can be formed into nearly ideal plane- or sphericalreferances and can then be assuned to scatter isotropically (i.e., s-1ike) and rery weakly with negligible seattexing phase shifts. some of the problens thet arise for the electron case and their potential solutions we briefly discuss below.

The reference wave an be highly anisotropic (e.g., p-like in s-level photounission), but at least it is best defined for the photoelectron case. In correcting for scattered-wave effects (see next paragraph), this anicotropy cen in principle also be divided out while doing the inageproducing transform.

- The scattered waves way be bighly anisotropic in anplitude and any show significant phase shifts due to scattering. Possible solutions to these probleas are to elininate or correct regions of the hologran that are wost anieotropic, as for example, over the forward scattering penks. For 
the forward-scattering case, Theruthasan et al. [44] have simply multiplied by a Gaussian-based damping function centered on a given forward scattering direction. Wore generally, it is also possible in principle to correct for anplitude and/or phase in doing the image-producing transform [47, 48]. One correction nethod proposed by Tonnex, saldin and co-workers [47] is simply to nornalize $\chi(\underline{k})$ by a generalized scattered-wave strength $F_{j}$ during the integration, which rields a new inage function $U$ ':

$$
U^{\prime}(x, y, z) \propto \iint\left\{x(\underline{k}) \exp \left[i k_{z} z\right] / F_{j}(\underline{k}, \underline{\underline{x}})\right\} \exp \left[i\left(k_{x} x+k_{y} y\right)\right] d k_{x} d k_{y} \mid \text {. }
$$

This has been termed the scattered-wave-included Fourier transform (SWIFI) method. In practice, this procedure has to date involved sinply dividing by a plane-wave or spherical-wave scattering factor, which aay then have to be adjusted with position in space so as to allow for the different types of scatterers present. The latter adjustment thus requires some advance knowledge of the structure, or an iterative approach. $F_{j}$ also can in principle allow for the anisotropy in the outgoing reference wave, as noted above, and the influence of such corxections have been quantitatively studied for Auger emission by Saldin, Barp, and Tonnex [11].

-The strength of the scattered waves may make self-interference terms of types (3) and (4) in Eq. 7 yield non-negligible inage features. This was first explored by Thevuthasan et al. [44], who found that such features should in nagnitude be only about 10-208 of the real or twin inages from terms (1) and (2) for a typical nedium-z scatterer. These effects have also been discussed by Bu et al. [45]. Thus, although interpreting weaker peaks in such holographic inages nay be complicated by self-interference effects, they should be negligible in first approxination. Suming several images obtained at different energies has in fact been shown to suppress such selfinterference problens [49], and we consider this correction nethod in nore detail below.

-In additional effect that has only recently been noted by Len et al. [50] is interference between the inages of different atons that have 
inversion symmetry along the surface with respect to the emitter (i.e., which are situated at $\pm r$ in the $x-y$ plane of the image). In fact, for certain choices of $|\underline{\underline{r}}|$ the phases of the two image features essentially cancel one another, and their amplitudes are thus found to oscillate with $|\underline{\mid}|$. This is illustrated in the theoretical simulations of Fig. 14 for a simple three-atom chain of No atoms consisting of one emitter and two scatterers at $\pm a$ along $x$. The oscillations predicted for an ideal weakscattering $x-r a y$ case is shown in (a), for an ideal weak-scattering electron case with only an s-wave scattering phase shift included in (b), and for full-strength scattering of electrons in (c). The $x$-ray and electron wavelengths are both fixed at $\lambda=0.621 \AA$, which corresponds to $x-r a y s$ at $19,970 \mathrm{ev}$ and electrons at $390 \mathrm{ev}$. For ideal scattexing in (a) and (b), the location of successive zeroes of order m can be predicted from the simple relation [50]: $a_{o} / \lambda_{e}=(2 m+1) / 4$; the same period is also found in (c) with full electron scattering, although with a slight phase shift and distortion of the curve shape. For full electron scattering, the complete extent of the effect is difficult to measure, as the real peak intensity becomes comparable to nearby satellite features. Thus, the curve in (c) is shown as dotted over the regions of minima. It is nonetheless clear that such interferences in electron scattering can lead to a suppression of the images by a factor of $<0.4$. Such interferences could thus accidentally reduce the images of certain near-neighbor features in holographic images, and minimally could make it non-trivial to quantitatively analyze the relative heights of different features. Analyzing only the region of the hologram dominated by one of the two atoms involved could assist in resolving this problen (see also discussion of this below in connection with real/twin overlap). Or suming images over several energies should also be useful in averaging over such effects, provided that the energies are chosen carefully so as to avoid sampling the interference zeroes preferentially [50].

-The overlap of real and twin images is a problem shared with optical holography, but it is potentially wure serious in images of surface structures, since the surface inherently breaks the inversion symetry along 
its normal, and thus the twins of substrate atoms may overlap the regions in space occupied by adsorbate or overlayer atoms. One solution to this problem is to note that, for some cases, the region of the hologram most strongly affected by some atom at $\underline{\underline{r}}$ is well localized in a solid-angle region centered on $\underline{\text {; }}$ this was first demonstrated in theoretical simulations by Saldin et al. [51]. Analyzing only this portion of the hologram then may lead to an image in which the twin from another atom at $-\underline{\underline{r}}$ is suppressed, as first suggested by Saiki et al. [28] A more general approach for suppressing twins has also been suggested by Barton [52], and it involves making phased summations of transforms obtained at different energies $E_{i}$ with wave vectors $k_{i}$ according to:

$$
u^{\prime \prime}(x, y, z) \propto \mid \Sigma_{i} \exp \left[-i k_{i} r\right] \iint\left\{\chi(\underline{k}) \exp \left[i k_{i z} z\right] / F_{j}(\underline{k}, \underline{r})\right\}
$$

This sum can in principle be performed either with or without correction for the scattered wave, although it has been included above in dividing again by $F_{j}(\underline{k}, \underline{r})$. In doing this sum, we have multiplied by the conjugate of the remaining phase factor due to path length difference exp[ikr], with $\chi(\underline{k})$ containing such factors inside of the $F_{j} \cdot s$ (cf. Egs. 2 and 3 ). The sum on $k_{i}$ now varies the magnitude of $k_{\text {, and }}$ selects out peaks at $r_{j}$ in space for

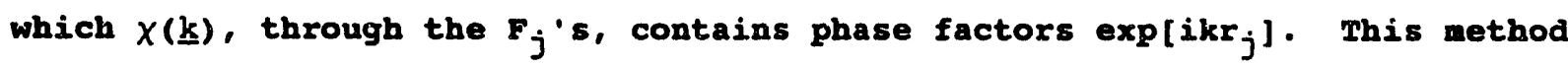
has been demonstrated to suppress twin images $[52,53]$, and also should suppress most effects due to multiple scattering [52], as discussed further below. Tong and co-workers $[48,54]$ have also proposed a somewhat different approach for analyzing multiple-energy data so as to simultaneously correct for scattered-wave effects and eliminate twin and multiple-scattering effects. This nethod does not require data sets over a large solid angle, but rather makes use of several scanned-energy diffraction curves that are then Fourier transformed and used to triangulate on the real-image positions of certain atoms [54].

- Multiple scattering effects also can effectively add in other pathlength differences that may in turn introduce spurious image features. As 
noted above, summing images over several energies appears to suppress such effects. Also, for some geometries, multiple scattering can be localized to one region of the hologram (e.g., along fairly dense chains of atoms with forward scattering at higher energies), and so by eliminating these regions from the analysis, these effects may also be suppressed.

-A final sort of problem is connected with the presence of more than one structurally-inequivalent type of emitter. If these cannot be resolved in the photoelectron/Auger electron spectrum, then the holographic images will be a superposition of the images around all of the emitter types, and this could seriously complicate interpretation. One common example of this is multilayer substrate emission (with at least one unique emitter type in each layer below the surface), for which the different superposed images correspond to varying degrees of both inelastic attenuation and multiple scattering. Emitters in each layer will generate an outgoing reference wave and thus also a hologram, but as the depth below the surface increases, thisi hologram will become weaker and more complex due to the combined effects of both inelastic scattering and multiple scattering. Thus, correcting for scattered wave and/or multiple scattering effects will become much more difficult due to the superposition of these holograms in the experimental data. For some problems, it will be possible with very high energy resolution to distinguish among the emitter types (as e.g., surface vs. bulk atoms and atoms in different oxidation states), and we have already noted that this is a key advantage of photoelectron diffraction. However, for other cases, it may make the interpretation of any holographic image much more difficult.

We now illustrate some of these difficulties and the effects of different correction procedures for a particularly simple model case: a linear 5-atom chain of Cu atoms $2.56 \mathrm{~A}$ apart 1 ying parallel to a fictitious surface along the $+x$ direction, with an emitter at one end of the chain that is also taken to be at the origin. A schematic drawing of the chain is shown at the top of Fig. 15, together with images derived in different ways. 
In (a), we begin with an ideal image from a hologram calculated with an $s$ outgoing wave and weak s-wave scattering: that is, a case that simulates an optical hologram but with wavelength appropriate to $1000 \mathrm{eV}$ electrons. This ideal image yields peaks essentially at the atomic positions, and identical twin images for negative $x$ (as it must). In (b), a fully accurate multiple scattering calculation for electrons at $1000 \mathrm{eV}$ has been used to generate the hologram, and a reasonably good image again is found, but now with shifts of the atomic peak positions to larger $x$ values by $0.20-0.35 \AA$, and again identical twin images for negative $x$. In (c), the hologram opening angle has been reduced from the full $180^{\circ}$ hemisphere above the surface (centered on the $z$ axis perpendicular to the chain) to $120^{\circ}$ in order to avoid regions in space where either strong forward scattering or multiple scattering can occur. This is found to improve the resolution of the peaks in the image, leading to reduced background and sharper features, and the peak shifts from the true atomic positions are also reduced somewhat to 0.10-0.20 A. In (d), the full hologram of (c) has been inverted according to Eq. 10 using the SWIFT procedure; the positions of the real image peaks are noticeably improved, now being within approximately $0.10 \AA$ of the true positions. However, the twin peaks are still rather strong, broader than before the correction, and shifted to even larger distances from the true positions. In fact, it is generally expected that twin images will be adversely affected in position, and often enhanced in intensity, by such scattered-wave corrections $[47,53]$. In (e), we consider a phased sum of image integrals according to Eq. 11, but without any scattered wave corrections. A total of 47 energies equally spaced in $\Delta k$ from 171.6 to 1000 eV have been used. For this summed-energy case, the twin images are indeed strongly suppressed, and the overall positions of the real peaks are about as good as the analogous single-energy image in (b). However, the summedenergy peaks are significantly broadened relative to those in (b), primarily due to the lower inherent resolution at the lower energies. Finally, in (f) we show a phased sum of image integrals over the sane set of energies, but with the scattered-wave correction applied to each integral according to Eq. 11. This yields the best image of all, in that the twins are still strongly 
suppressed and the real peaks are moved to within about $0.05-0.10$ of the true positions.

Thus, there is clearly a hierarchy of levels at which such data can be taken and the analysis carried out. It seems clear however, that, without some sort of correction for scattered-wave effects, the images can be significantly shifted from the atomic positions. Summing energies also looks very promising, even though it increases the net amount of data taking by about an order of magnitude. In fact, it has been verified that only about 10 energies is sufficient to yield most of the benefits of this kind of approach [49]. Some multi-energy experiments have already been carried out with existing synchrotron radiation facilities [55], and the analyses of these data verify the suppression of twin images. Such experiments will be well-suited to the next-generation high-brightness sources that are just beginning to come on line.

\subsection{Some applications to experimental data:}

To date, there are relatively few examples of suitable experimental data that have been analyzed holographically. Most of the cases studied have been for multilayer substrate emission, a situation for which we have previously noted that the several types of distinct images expected may well complicate both the analysis and the overall ability to derive useful structural information. For example, in the first holographic images for Kikuchi backscattering and Auger emission from Cu(001) due to Barp, Saldin, and Tonner [42,43], certain planar cross sections clearly showed features associated with the nearest-neighbor atoms to a typical emitter in the fcc lattice. However, these images were elongated parallel to strong forward scattering directions (e.g., the <110> directions), and it is unclear that any new information concerning the radial position of the nearest neighbors was present in these images. Subsequent work by Herman et al. on si 2p emission from si(111) [14] showed similar results: features associated with next-nenrest neighbors were clear in the images, but again with significant 
elongation parallel to forward scattering directions. Tran et al. found essentially identical results for Ge 3p emission from Ge(111) [31]. Thus, it is suggested that holographic analyses may be more fruitfully carried out on very thin overlayers, as e.g., the system Cu on Pd(111) studied by Hardcastle et al. [56], or on adsorbate layers.

As one example of an adsorbate which also illustrates the use of several of the image correction procedures mentioned above, we consider again the well-defined test case of $c(2 \times 2) 5 / \mathrm{Ni}(001)$ (cf. the trial and error analysis of this structure in Fig. 8). Thevuthasan et al. [57] have recently studied $s 2 p$ emission from this system at $1327 \mathrm{eV}$ kinetic energy. A novel aspect of this work was to take data over a range of takeoff angles relative to the surface from $10^{\circ}$ to $40^{\circ}$ so as to optimize the quality of the image in several respects: by starting only at $10^{\circ}$ but not going lower, effects due to both forward scattering and multiple scattering along the surface are largely avoided; by going up to $40^{\circ}$ (or even higher if intensity had permitted) the data range included the highest degree of diffraction anisotropy or largest oscillations in $\chi(\underline{k})$; and by using a $\underline{k}$ range over which $\Delta k_{x}$ and $\Delta k_{y}$ (parallel to the surface) were of about the same magnitude as $\Delta \mathrm{k}_{\mathrm{z}}$ (perpendicular to the surface) the resolution in all three coordinates could be expected to be more nearly equal than in several prior studies for which $\Delta \mathbf{k}_{\mathrm{x}}=\Delta \mathrm{k}_{\mathrm{y}} \gg \Delta \mathrm{k}_{\mathrm{z}}$. (See earlier discussion of Eq. 9.) Two other image improvement methods also were tested: the SWIFT procedure was used to correct for scattered-wave effects, and only one half of the hologram (toward $+x$ ) was analyzed in order to focus on the real image of the nearest-neighbor $s$ atom along $+x$ and avoid undesirable real/twin overlap during the correction and imaging procedure. Some of the results of this study are shown in Fig. 16, where the top two panels are based on experimental data and the bottom two are based on a single-scattering simulation of the same hologram. (For the $10^{\circ}-40^{\circ}$ range of takeoff angles in this data, it has been verified that single scattering is a very accurate approximation.) Even before any scattered-wave correction (panels (a) and (c)), the nearest-neighbor $\mathbf{s}$ images are very clear in both experiment and 
theory, although in both cases they are shifted by about the same amount (0.6-0.7 $\AA$ ) to larger $x$ values than the known position. After applying the SWIFT procedure however (panels (b) and (d)), both experiment and theory are improved significantly in position, now being within $0.2 \AA$ of the true positions. Note that the twins again behave in the opposite sense in these images upon correction (cf. Fig. 15). The next-nearest-neighbor images are not seen for experiment, although they are strong in theory; perhaps this is due to statistical scatter in the experimental data, and if so one could hope for better resolution of them in future work.

Although not shown here, it was also found that the resolution of the nearest-neighbor features in both experiment and theory was approximately equal in all of $x, y$, and $z$. Thus, this method of taking and analyzing higher-energy photoelectron holography data appears very promising for adsorbate studies. Especially if combined with phased sums over several energies, such images could be accurate to within a few tenths of an Angstrom, and be essentially free of tir in interferences.

Finally, we note that, in view of the several problems encountered with such holographic imaging, it seems unlikely that such images will ever in a single step yield structures with the accuracy and detail discussed in Sec. 3.1. However, holography could be extremely useful in quickly establishing site symmetries and approximate structures that could be refined by the more laborious trial-and-error methods, but with much less time and much less probability of falling into a false structure that is not the global best fit to the data. Thus, although there are still many questions to be answered as to how well this new direction will work out, it certainly has sufficient promise to be pursued vigorously.

\subsection{Application to short-range magnetic order:}

We have previously noted in discussing Fig. 11 that spin-up and spindown electrons in a magnetically-ordered system will exhibit measurable 
differences in their scattering processes, especially if observations are made at lower energies of 50-100 ev. The question thus immediately axises as to whether a holographic analysis of spin-polarized diffraction data could be used to directly study short-range magnetic order. Although there is as yet no experimental data on this subject, Raduwela et al. [58] have carried out model calculations on some simple clusters to ascertain whether this is feasible. Some of their results are shown in Fig. 17 for a two-atom cluster in which one $\mathrm{Mn}^{2+}$ ion is the emitter and the other is a magnetically-ordered scatterer. In order to look for spin-dependent exchange effects in the scattering, images $U(x, y, z, \uparrow)$ and $U(x, y, z, t)$ were calculated from Eg. 9 for outgoing spin-up and spin-down electrons, respectively; no scattered-wave correction was used in order to focus on the spin-dependent differences in the images. The kinetic energy was held constant at $120 \mathrm{eV}$ for both cases. The exchange interaction with the five parallel-coupled 3d electrons (cf. Fig. 11(a)) was included in the scattering potential if the photoelectron spin was parallel to the net spin of the $\mathrm{Mn}^{2+}$ scatterer, and was omitted if the photoelectron spin was antiparallel to the scatterer spin. Thus, any difference between the spinup and spin-down images should be sensitive to the orientation of the scatterer. A suitable measure of this difference is found to be:

$$
\Delta(x, y, z, \uparrow-\downarrow)=U(x, y, z, \uparrow)-U(x, y, z, \downarrow),
$$

and it is plotted along with the two $U$ 's for two different orientations of the scatterer in parts (a) and (b) of Fig. 17. The boldface arrows in the arguments in this figure here represent the orientation of the scatterer spin. In part (a) for a scatterer with moment parallel to that of the emitter, the two U's are indeed slightly different, but it is only on looking at $\Delta$ that a clear dip just at the position of the $\mathrm{Mn}^{2+}$ scatterer is seen. This dip is furthermore about 108 of the full heights of the $U$ functions in magnitude, and thus is in principle measurable. In part (b) for a scatterer that is anti-parallel to the emitter, certain symmetries of the interactions with respect to the electron and scatterer spins dictate 
that the two $U$ curves just reverse notation, and that the dip in $\Delta$ in (a) thus becomes a peak in (b). Thus, a real near-neigbbor spin flip (e.g., as temperature is changed) could produce about a $20 \%$ change in the difference image $\Delta$ that again should be measurable. In an independent study by Timmermans et al. [59], a slightly different sort of experiment involving the use of an external spin detector and another difference function of the form $\Delta^{\prime}=|F(x, y, z, \uparrow)-F(x, y, z, \downarrow)|$, where $F$ is the (complex) Fourier transform integral within $U$, has been proposed. Such measurements also should be of interest, although they would be considerably more difficult due the approximately $10^{4}$ times greater data acquisition times in the external spin detector. Also, in tests of $\Delta$ versus $\Delta^{\prime}$ on the cases of Fig. 17, $\Delta$ has been found to be a much more sensitive indicator of spin-dependent scattering $[58]$.

Thus, these preliminary theoretical results suggest that the direct imaging of short-range spin order might also be possible through a holographic analysis of spin-polarized photoelectron diffraction data. The difference function $\Delta$ used here also seems to have the desirable feature that it yields peaks which are better centered on the atomic positions than those in the initial images $U$. Perhaps this simplification arises due to the perturbative nature of the exchange scattering, although further tests of this for more complex clusters are called for. We also note that this is not a case where carrying out a full correction for scattered-wave effects is useful, as we in fact wish to measure a difference in the scattering of the two types of electrons. Although taking such data would again require high-intensity synchrotron radiation, it has some very interesting prospects for application to magnetic surfaces, overlayers, and nanostructures.

\section{ACKNOWLEDGMENTS:}

The work discussed here that has been performed by the author's group has been supported by the office of Naval Research undex Contract N00014-90J-1457 and by the U.S. Department of Energy under Contract DOE-ACO3- 
765F00098, as well as by grants of computing time at the San Diego Supercomputer Center. A.P. Kaduwela, S. Thovuthasan, and T.T. Tran are also gratefully acknowledged for belpful comments conceraing this manuscript.

\section{REFERENCES:}

[1] C.S. Fadley, Prog. in Surf. Sci. 16, 275 (1984).

[2] (a) C.S. Fadley, Pkys. Scripta T17, 39 (1987); (b) C.S. Fadley, A.I.P. Conference Proceedings No. 215 (Amer. Inst. of Phys., New York, 1990).

[3] S.A. Chambers, I.M. Vitomirov, S.B. Anderson, B.W. Chen, T.J. Wagner, and J.H. Weaver, Superlatt. and Microstruct. 3,563 (1987); S.A. Chambers, Adv. in Phys. 40, 357 (1990); S.A. Chambers, Surf. Sci. Repts. 16, 261 (1992)--The botton two panels of Fig. 31 in this last review have been corrected in the final version of this work appearing in $s$. Thevuthasan et al., J. Vac. Sci. Technol. A10, 2261 (1992).

[4] W.F. Egelhoff, Jr. in Critical Reviews in Solid state and Materials Sciences, 16,213 (1990).

[5] C.S. Fadley, in Synchrotron Radiation Research: Advances in Surface Science, edited by R.Z. Bachrach (Plenum, New York, 1992).

[6] A. Szöke, in Short Wavelength Coherent Radiation: Generation and Applications, edited by D.T. Attwood and J. Boker, AIP Conference Proceedings No. 147 (AIP, New York, 1986).

[7] (a) J.J. Barton, Phys. Rev. Lett. 61, 1356 (1988); (b) J.J. Barton, J. Electron spectrose. 51, 37 (1990). 
[8] P. Alnot, J. Olivier, F. Wyczisk, and C.S. Fadley, J. Electron Spectrosc. 43, 263 (1987); P. Alnot, J. Olivier, and C.S. Fadley, J. Electron Spectrose. 49,159 (1989).

[9] (a) C.H. Ii and S,Y. Tong, Phys. Rev. Lett. 42, 901 (1979); (b) J.J. Barton and D.A. Shirley, Phys. Rev. B32, 1892 (1985); and Phys. Rev. B 32 , 1906 (1985); (c) A.P. Raduwela, G.S. Herman, D.J. Friedman and C.S. FadleY, Phys. Scripta 4I, 948 (1990); (d) A.P. Kaduwela, D.J. Friedman, and C.S. Fadley, J. Electron Spectrosc. 57,223 (1991).

[10] S.M. Goldberg, C.S. Fadley, and S. Kono, J. Electron Spectrosc. 21,285 (1981); s.y. Tong and C.B. Ii, in Critical Reviews in solid state and Materials Sciences edited by D.E. Schuele and R.W. Hoffman (CRC Press, Cleveland, 1981) Vol. 1.

[11] D.K. Saldin, G.R. Harp, and B.P. Tonner, Phys. Rev. B45, 9629 (1992) and references therein.

[12] S.Y. Tong, H.C. Poon, and D.R. Snider, Phys. Rev. B 32 , 2096 (1985).

[13] M.I. Xu and M. Van Eove, Surf. Sci. 207, 215 (1989).

[14] [9] G.S. Herman, S. Thevuthasan, Y.J. Kim, T.T. Tran and C.S. Fadley, Phys. Rev. Lett. $\underline{68}, 650$ (1992).

[15] (a) D. Naumovic, A. Stuck, T. Greber, J. Osterwalder, and I. Schlapbach, Phys. Rev. B, in press; (b) A. Fischer, R. Fasel, J. osterwalder, A. Krozer, and I. Schlapbach, to be published.

[16] P.J. Orders and C.S. Fadley, Phys. Rev. B27, 781 (1983); M. Sagurton, E.I. Bullock, and C.s. Fadley, Phys. Rev. 30, 7332 (1984).

[17] (a) J.B. Pendry, Low Energy Electron Diffraction (Academic Press, London, 1974); (b) M.A. Van Hove and S.Y. Tong, Surface Crystallography by 
IEED (Springer-Verlag, New York, 1979) (c) M.A. Van Hove, W.H. Weinberg, and C. $-M$. Chan, in Low Energy Electron Diffraction: Experiment, Theory, and Surface structure Determination, Ed. G. Ertl (Springer-Verlag, Berlin, 1986) pp. 246-248.

[18] R. Beinz, U. Starke, and F. Bothe, Surf. Sci. Lett. 243, L70 (1991); R. Heinz, U. Starke, M.IA. van Hove, and G.A. Samorjai, Surf. Sci. Lett. 261. 57 (1992); K. Heinz, private communication of results for $\mathrm{K} / \mathrm{Ni}(001)$.

[19] I.G. Petersson, S. Rnno, N.F.T. Hall, C.S. Fadley, and J.B. Pendry, Phys. Rev. Lett. 42,1545 (1979); P.J. Orders, S. Kono, C.S. Fadley, R. Trehan, and J.T. Lloyd, Surí. Sci. 119, 371 (1982).

[20] D.A. Wesnen, F.P. Coenen, and H.P. Bonzel, Phys. Rev. Lett. 60. 1045 (1988); and Phys. Rev. B $\underline{39}, 10770$ (1989); and H.P. Bonzel, review to appear in Prog. in Surf. Sci.

[21] R.S. Saiki, G.S. Herman, J. Osterwalder, M. Yamada, and C.S. Fadley, Phys. Rev. Lett. 63,283 (1989).

[22] A. Nilsson, H. Tillborg, and N. Mårtensson, Phys. Rev. Lett. 67, 1015 (1991).

[23] G.S. Herman, D.J. Friedman, T.T. Tran, C.S. Fadley, G. Granozzi, G.A. Rizzi, J. Osterwalder, S. Bernardi, J. Vac. Sci. Technol. Bg, 1870 (1991); G.S. Herman, Ph.D. Thesis (University of Hawaii, 1992) .

[24] (a) R.S. Saiki, A.P. Kaduwela, J. Osterwalder, C.S. Fadley, and C.R. Brundle, Phys. Rev. BLQ, 1586 (1989); (b) R.S. Saiki, A.P. Kaduwela, J. Osterwalder, D.J. Friedman, C.S. Fadley, and C.R. Brundle, Surf. Sci. 279, 305 (1992)..

[25] D. Sebilleau, M.C. Desjonqqueres, D. Chaveau, C. Guillot, J. Lecante, G. Treglia, and D. Spanjaard, Surf. Sci. Lett. 185, L527 (1987). 
(26) (a) F.J. Eimpsel, F.R. Wcreely, A. Talab-Ibrahin, J.A. Yaxnoff, and G. Eollinger, Phys. Rev. B38, 6084 (1988); (b) J.D. Danlinger, E. Rotenberg, U. Dessinger, M. Leskovar, and M.A. Olmstead, private commication.

[27] I.-Q. Wang, 8. Eussain, 2.Q. Eunag, A.z. Schach von Wittenau, D.A. Shirley, and D.W. Lindle, Phys. Rev. B44, 13771 (1991).

[28] R.8. Saiki, A.P. Taduwela, Y.J. Kie, D.J. Eriednan, J. Osterwalvsr, s. Theruthasan, and C.s. Fadley, Surf. Sci. 282, February 10, I993, in press.

[29] L.J. Ternine].10, X.5. Itang, Z.Q. Eunng, S. Kin, M.E. Schnch von Wittearu, K.I. Leung, and D.h. EkirleY, Phys. Rev. B38, 3879 (1988).

[30] U. Breuer, E.P. Bonzel, K.C. Prince, nd R. Lipowsky, Surf. Sci, 23, 258 (1989); U. Brever, O. Knavff, and H.P. Bonzel, J. Vac. Sci. Technol. AB, 2489 (1990).

[31] I.T. Iran, 8. Theruthesan, Y.J. Kin, G.8. Bernan, D.J. Friednan, and C.8. Fadley, Phys. Rer. B45, 12106 (1992): and surf. sci., to appear.

[32] E.6. Menae and R. Malic, Phys. Rev. Lett. 58, 1437 (1987); and Pbys. Rev. B.38, 13163 (1988).

[33] 8.A. Chambers, J. Vac. Sci. Technol. A, 2459 (1989).

[34] A.W. Danier van der Con, J.H. CaY, J.H.M. Frenken, and J.F. van der Veon, surf. Bci. 241, 335 (1991).

[35] B. Sinkovic and C.s. Fadley, Prys, Rev. B31, 4665 (I985).

[36] (a) B. Einkoric, B.D. Eernsecier, and C.8. FadleY, PhYs. Rev. Lett. 55, 1227 (1985); (b) B. Sinkovic, D.J. Friednan, and C.8. Fadley, J. Magn. Uagn. vater. 92,301 (1991). 
[37] B.D. Hernsmeier, J. Osterwalder, D.J. Friednan, and C.S. Fadley, Phys. Rev. Lett. 62, 478 (1989); B.D. Hexasmeier, J. Osterwalder, D.J. Friedman, B. Sinkovic, T.T. Tran, and C.S. Fadley, Phys. Rev. B42, 11895 (1990).

[38] 2. Hussain, C.S. Fadley, and S. Kono, Phys. Rev. B22, 3750 (1980); R.C. White, C.S. Fadley, M. Sagurton, and 2. Bussain, Phys. Rev. B34, 5226 (1986).

[39] J. Osterwalder, T. Greber, A. Stuck, and I. Schlapbach, Phys. Kev. Lett. 64, 2683 (1990).

[40] G.S. Benan, T.T. Tran, K. Bigashiyama, and C.S. Fadley, Phys. Rev. Lett. 68,1204 (1992).

[41] A. Stuck, J. Osterwalder, T. Greber, S. Buefner, and I. Schlapbach, Phys. Rev. Lett. 65, 3029 (1990).

[42] G.R. Barp, D.X. Saldin, and B.P. Tonner, Phys. Rev. Lett. 65, 1012 (1990).

[43] G.R. Barp, D.K. Saldin, and B.P. Tonner, Phys. Rev. B42, 9199 (1990).

[44] S. Thevuthasan, G.S. Berman, A.P. Kaduwela, R.S. Saiki, Y.J. Kim, W. Nienczure, M. Burger and C.S. Fadley, Phys. Rev. Lett. 67, 469 (1991).

[45] P. Bu, C.J. Barnes, and D.A. King, Chem. Phys. Lett. 183, 521 (1991).

[46] (a) D.x. Saldin and P.I. de Andres, Phys. Rev. Lett. 64, 1270 (1990);

(b) M.A. Mendez, C. Gluck, and K. Beinz, J. Phys.: Cond. Mat. 4, 999 (1992).

[47] (a) B.P. Tonner, Z.-L. Zan, G.R. Barp, and D.X. Saldin, Phys. Rer. B43, 14423 (1991); (b) G.R. Barp, D.X. Saldin, X. Chen, Z.I. Ean, and B.P. Tonner, J. Electron spectrosc. 258, 313 (1991). 
[48] S.Y. Tong, C.M. Wei, T.C. zhao, E. Buang, and B. Li, Phys. Rev. Lett. 66, 60 (1991).

[49] S. Theruthasan and C.S. Fadley, to be published.

[50] P. Ien, S. Theruthasan, and C.S. Fadley, to be published.

[51] D.K. Saldin, G.R. Barp, B.L. Chen and B.P. Tonner, Phys. Rev. B 44,2480 (1992)

[52] J.J. Barton, Phys. Rev. Lett. 67, 3106 (1992).

[53] S. Thevuthasan, G.S. Herman, A.P. Kaduwela, T.T. Tran, Y.J. Kim, R.S. Saiki, and C.S. Fadley, J. Vac. Sci. Technol. A110, 2261 (1992).

[54] S.Y. Tong, H. Li, and B. Buang, Phys. Rev. Lett. 67, 3102 (1992); S.Y. Tong, A. Euang, and C.M. Wei, Phys. Rev. B46, 2452 (1992).

[55] I.J. Texminello, J.J. Barton, and D.A. Lapiano-Smith, J. Vac. Sci. Technol. B10, 2088 (1992) and private comunication; E. Petersen, I. Terminello, J.J. Barton, and D.A. Shirley, private comunication.

[56] S. Eardcastle, 3.-I. Ean, G.R. Barp, J. Zhang, B.I. Chen, D.K. Saldin, and B.P. Tonner, Surf. Sci. 245 , L190 (1991).

[57] S. Thevuthasan, R.X. Ynzunza, E.D. Tober, C.S. Fadley, A.P. Raduwela, and K.A. van Bove, Phy . Rev. Lett., in press.

[58] A.P. Kaduwela, 2. Wang, S. Theruthasan, H.A. Van Eove, and C.S. FadleY, to be published.

[59] E.M.E. Timemans, G.T. Tramel1, and J.P. Bannon, to be published. 


\section{FIGURE CAPTIONS:}

Fig. 1- Illustration of the basic process involved in photoelectron diffraction, with various important physical variables indicated. Only single scattering is indicated for simplicity. In Auger electron diffraction, either a photon or an electron may initiate the two-step emission process. In a holographic interpretation of such measurements, the direct or unscattered wave $\phi_{0}$ is identified with the reference wave, and the scattered waves $\phi_{j}$ are identified with the object waves.

Fig. 2- Experimental and theoretical si 2p diffraction patterns above a Si(111) surface, as obtained over most of the full $2 \pi$ solid angle above the surface and then projected down onto the $k_{x}-k_{y}$ surface plane. The kinetic energy is $1388 \mathrm{eV}$. The contours have been normalized by subtracting the unscattered intensity $I_{0}$ and then adjusting the vertical scale so as to optinally match the features away from the lowest-index forward scattering directions (which are labelled). Note the generally excellent agreenent between experiment and theory away from these directions, and the overestinate of forward scattering along them in a single-scattering picture [Fron ref. 14]

Fig. 3- (a) Experimental polar scans in two high-symmetry azimuths of the intensity ratio I(C1s)/I(01s) for the tilted $\alpha_{3}$ state of co on Fe(001). The electron energies are 1202 eV for C 18 and 945 ev for 0 1s. (b)

Experinental azimuthal scan of C 18 intensity at a takeoff angle with respect to the surface of $35^{\circ}$. (c) The bonding geometry deduced from these data. [Froa ref. 21]

Fig. 4- 18 core spectra for two polar angles of enission from $\mathrm{N}_{2}$ on $\mathrm{Ni}(001)$, showing the two chemically-shifted components $N$ (bound to Ni) and $N^{\prime}$ (bound to only $N$ ). The angle $\theta$ is here measured relative to the surface normal. The kinetic energies are about $1086 \mathrm{ev}$. In the inset at upper 
left, the intensity ratio $I(N) / I\left(N^{\prime}\right)$ is plotted as a function of polar angle. [From ref. 22]

Fig. 5- Azimuthal diffraction curves for $\mathrm{Bg}, \mathrm{Cd}$, and Te emission from a $\mathrm{Hg}_{1-}$ $x^{C d}$ TE surface with (111) oxientation. In (a), full-symetry experimental scans over more than $120^{\circ}$ are compared to the results of single-scattering calculations for the two possible terminations of the surface: $A$ = cationic and $B=$ anionic. R-factors have been calculated between experiment and theory also. In (b), the strong nearest-neighbor forward scattering peaks that are expected to be overestimated in intensity in single scattering have been eliminated, and the curves renormalized to one another over the middle $\phi$ range. [From ref. 23]

Fig. 6- Mg 15 diffraction patterns from Mg deposited on Pd(111). The electrons have been excited with $\mathrm{Si} K \alpha \mathrm{x}$-rays and have a kinetic energy of $437 \mathrm{eV}$. Note the early onset of forward scattering peaks at only $0.5 \mathrm{~kL}$ coverage, and the change in symmetry of the pattern from threefold to sixfold between 0.9 and $1.5 \mathrm{kr}$. [From ref. $15(\mathrm{~b})$ ]

Fig. 7- Experiment and multiple-scattering theory for scanned-energy Cl 15 photoelectron diffraction from $(\sqrt{3} \times \sqrt{3}) R 30^{\circ}$ on $N i(111)$. The kinetic energy range was 50 to $550 \mathrm{eV}$. In (a), a $0.1 \AA$ or 58 contraction in the distance between the first and second $N i$ layers has been incorporated, and in (b), the distance between these two layers has been left at the bulk value. [From ref. 27]

Fig. 8- Experiment and single-scattering theory for azimuthal scanned-angle $x$-ray photoelectron diffraction from $c(2 \times 2) S$ on Ni(001). (a) R-factors sumed over data for takeoff angles of $\theta=6^{\circ}, 10^{\circ}, 12^{\circ}$, and $16^{\circ}$ and plotted versus $s$ vertical position for various distances $d_{12}$ between the first and second Ni layers. (b) Direct comparison of experimental and theoretical diffraction curves for the optimum geometry of $z=1.39 \AA$ and $d_{12}=1.86 \AA$. Multiple scattering effects are thought to be responsible for the greater discrepancies seen at the lowest takeoff angle of $6^{\circ}$. [From ref. 28] 
Fig. 9- Different types of experimental results concerning a hightemperature surface disordering transition on Ge(111), with a common reference temperature of $1060 \mathrm{~K}$ indicated by the vertical dashed line in each panel. (a) The intensity of the (11) LFED beam at an $80 \mathrm{eV}$ incident energy as a function of temperature. The filled square points represent the maximum peak height minus background, and the open circles the peak area, again minus background. [From ref. 32] (b) The photoelectron intensity in a nearest-neighbor forward scattering peak along a $\langle 11,-1\rangle$ direction for Ge $3 p$ emission at about $1364 \mathrm{eV}$ as a function of temperature. The takeoff angle is $\theta=19^{\circ}$. The intensity is measured as a peak height minus background in an azimuthal scan. [From ref. 31] (c) The number of disordered surface layers as determined from photoelectron diffraction data at two different photoelectron takeoff angles of $19^{\circ}$ and $55^{\circ}$ are compared to analogous numbers derived from medium energy ion scattering data at three different energies of $60 \mathrm{kev,} 100 \mathrm{kev,} \mathrm{and} 175 \mathrm{keV}$. All values are normalized to 100 at their high-temperature maxima. [From refs. 31 and 34]

Fig. 10- Full hemisphere diffraction patterns for Ge 3p emission at 1364 eV from Ge(111), as projected down onto the $k_{x}-k_{y}$ surface plane. Data are shown for three temperatures: (a) room temperature, (b) $970 \mathrm{~K}$ just below the disordering transition, and (c) $1130 \mathrm{~K}$ just above this transition. Note the strong similarity of this data to that in Fig. 2. [From ref. 31]

Fig. 11- (a) Multiplet-split Mn 38 core spectra in an $x$-ray photoelectron spectrum from $\mathrm{KanF}_{3}$, with the I-s coupling origins of the two peaks and their predominant spin orientations with respect to the net $3 d$ spin of the emitting $\mathrm{Mn}^{2+}$ ion indicated. (b) The experimental temperature dependence of the normalized spin-up/spin-down intensity ratio of the two multiplet peaks in (a) for emission along two directions above a $\operatorname{din}_{3}(110)$ surface and at a lower kinetic energy of about $104 \mathrm{ev}$. The ratio at a given measurement temperature "IT" has been normalized to the ratio at the maximum temperature of the experiment "BI" according to the formuja in the inset. Note the 
sharp change in this ratio at a temperature that is about $2.7 \times$ the longrange-order transition temperature (Neel temperature, $T_{N}$ ). [From ref. 36 (a) ]

Fig. 12- X-ray photoelectron spectra from the valence bands of W(110) are shown as a function of azimuthal angle for a constant polar angle of $45^{\circ}$ with respect to the surface. All spectra have been normalized to the same maximum height for the strongest peak at $2.8 \mathrm{eV}$. In (a), the temperature is $295 \mathrm{~K}$ and about $55 \%$ of the transitions are estimated to be direct. In (b), the temperature is $803 \mathrm{~K}$, and only about $20 \%$ of the transitions should be direct. [From ref. 40]

Fig. 13- X-ray photoelectron intensities from the valence bands of $W(110)$ as energy-integrated over both of the features seen in Fig. 12 are compared with the $4 f$ core intensities from the same surface. In (a), the temperature is $295 \mathrm{~K}$, and in (b) it is $803 \mathrm{~K}$. Normalized ratios of the valence and core intensities are also shown in the lower portion of each panel. [From ref. 401

Fig. 14- Predicted oscillations with bond distance $; 1$ the Fourier-transform holographic peak amplitudes for a simple three-atoin chain of Mo atoms with one central emitter and two scatterers symmetrically arranged at distances of ta from the emitter along the x-axis. Single-scattering calculations are shown at a fixed wavelength of 0.620 for: (a) essentially ideal $x-r a y$ scattering, (b) nearly ideal electron scattering in which only the $1=0$ phase shift has been used, and (c) full electron scattering. The orders of interference between the images at $+a$ and $-a$ expected on the basis of the simple equation given in the text are also indicated. [From ref. 50]

Fig. 15- Fourier transform magnitudes from multiple scattering $\chi(\underline{k})$ 's as calculated along the axis of a 5-atom horizontal Cu chain. An s-wave $\phi_{0}$ is assumed as outgoing from the end atom of the chain and the electron energy is $1000 \mathrm{ev}$. (a) Ideal weak s-wave scattering and with a hologram opening 
angle of $178^{\circ}$. (b) Full electron scattering strength and an opening angle of $178^{\circ}$. (c) As (b), but with a reduced opening angle of $120^{\circ}$. (d) As (b), but after scattered-wave corrections by the SWIF"m method. (e) Phased summation of FT's for 47 energies between 171 . Ev and $1000 \mathrm{eV}$, again with opening angle of $178^{\circ}$. (f) As (e), but for a phasted sum of swrFr-corrected transforms. [From ref. 53]

Fig. 16- Fourier transform contour plots in the $x-y$ surface plane for $s$ 2p emission from $c(2 \times 2) S$ on $\mathrm{Ni}(001)$. This plane cuts through all of the $\mathrm{s}$ scatterers. Only the right half of the hologram has been analyzed to minimize real/twin overlap, and the swIFT scattered-wave correction procedure has been used in (b) and (d). Experimental results have been used to derive the images in (a) and (b), and single-scattering theory in (c) and (d). [From ref. 57]

Fig. 17- Holographic image functions $U$ and (spin-up) - (spin-down) difference functions $\Delta$ for a cluster of two $\mathrm{Mn}^{2+}$ ions consisting of an emitter and scatterer that are $4.0 \AA$ apart. The photoelectron energy is 120 ev for both spin-up and spin-down photoelectrons. (a) scatterer spin up. (b) Scatterer spin down. [From ref. 58] 


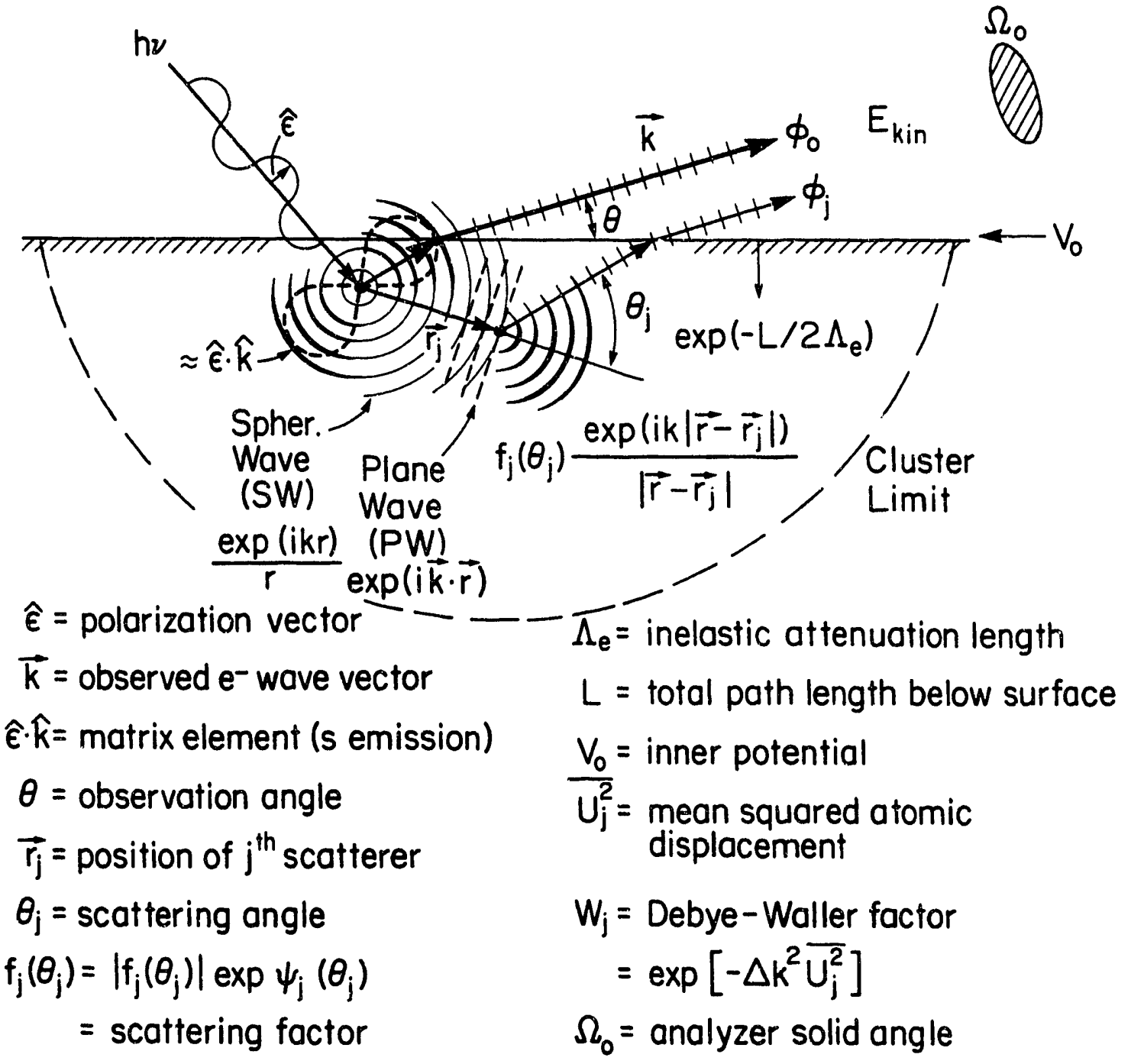




\section{Si(111): Si $2 p$ emission at $1388 \mathrm{eV}$}
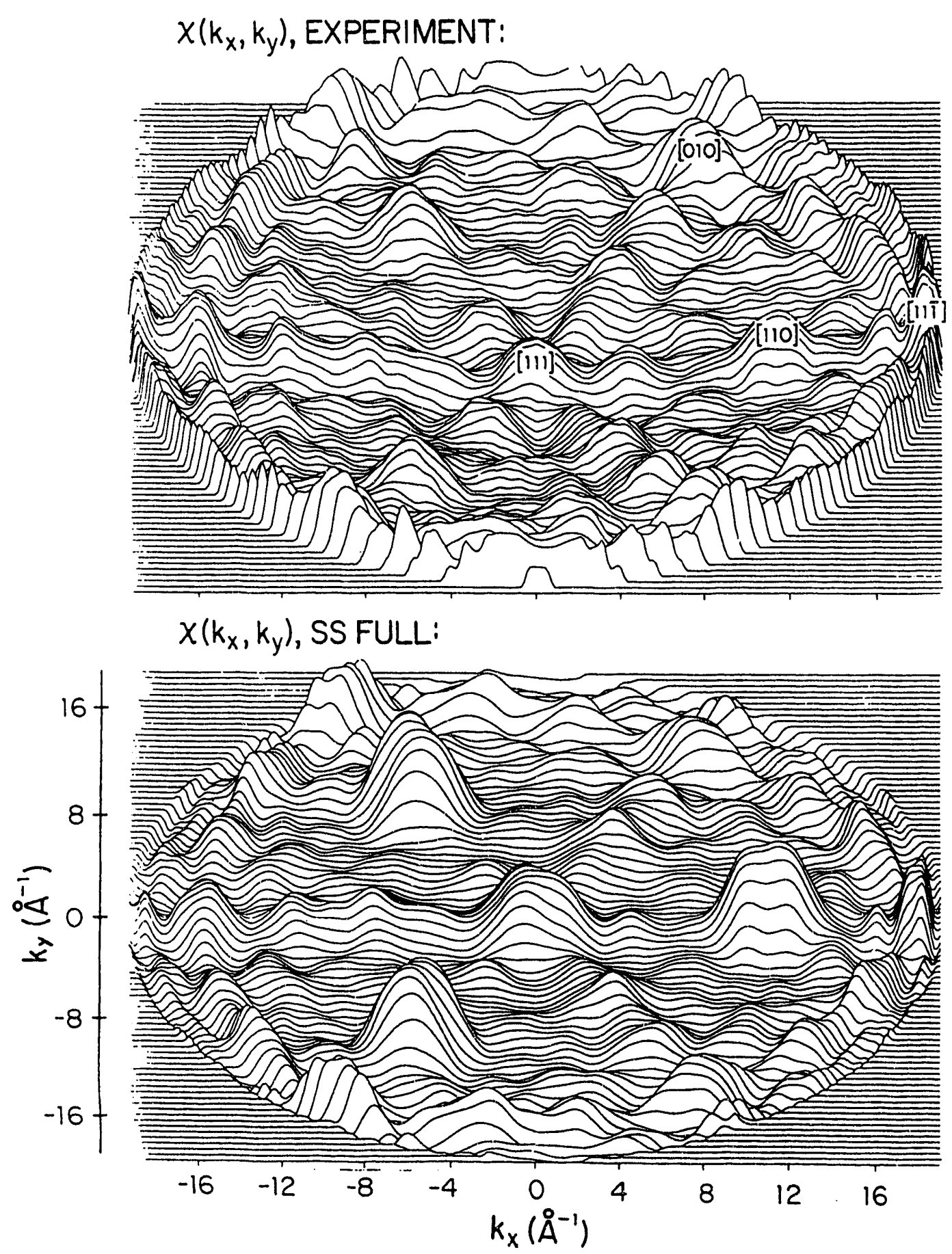

XBL $9211-2481$

\section{Figure 2}



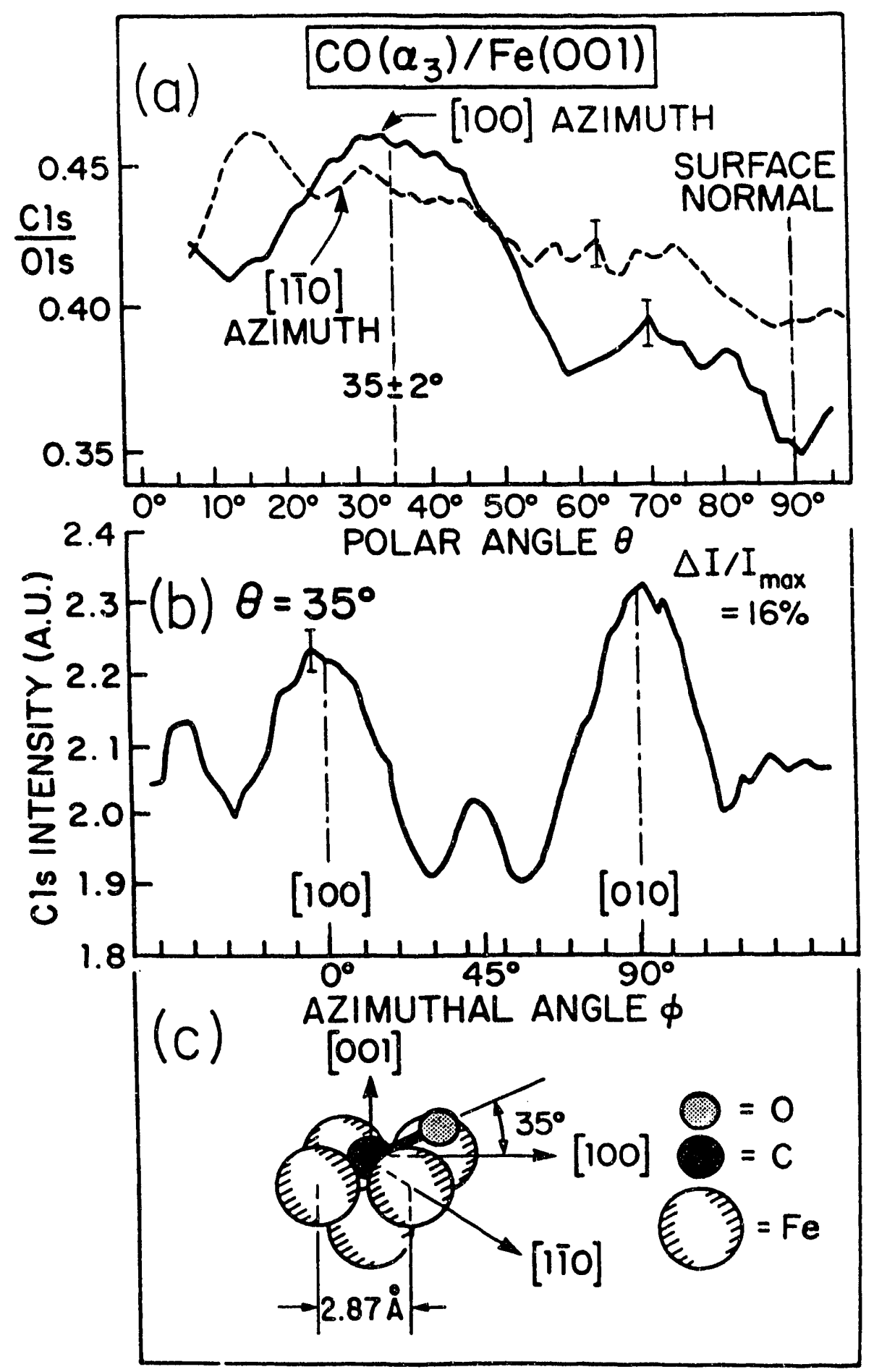

XBL $9211-2482$

Figure 3 


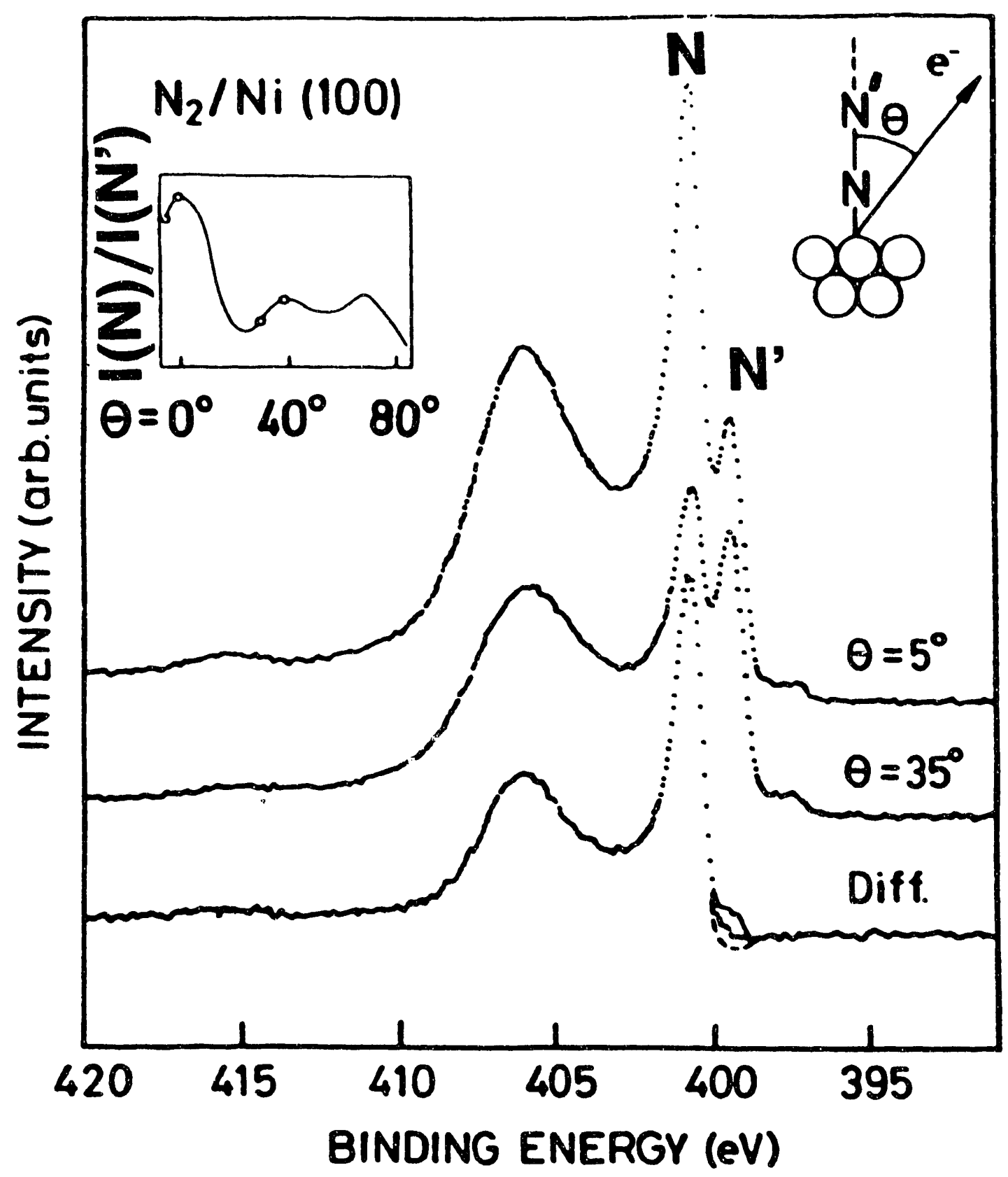

XBL 9211-2490

Figure 4 


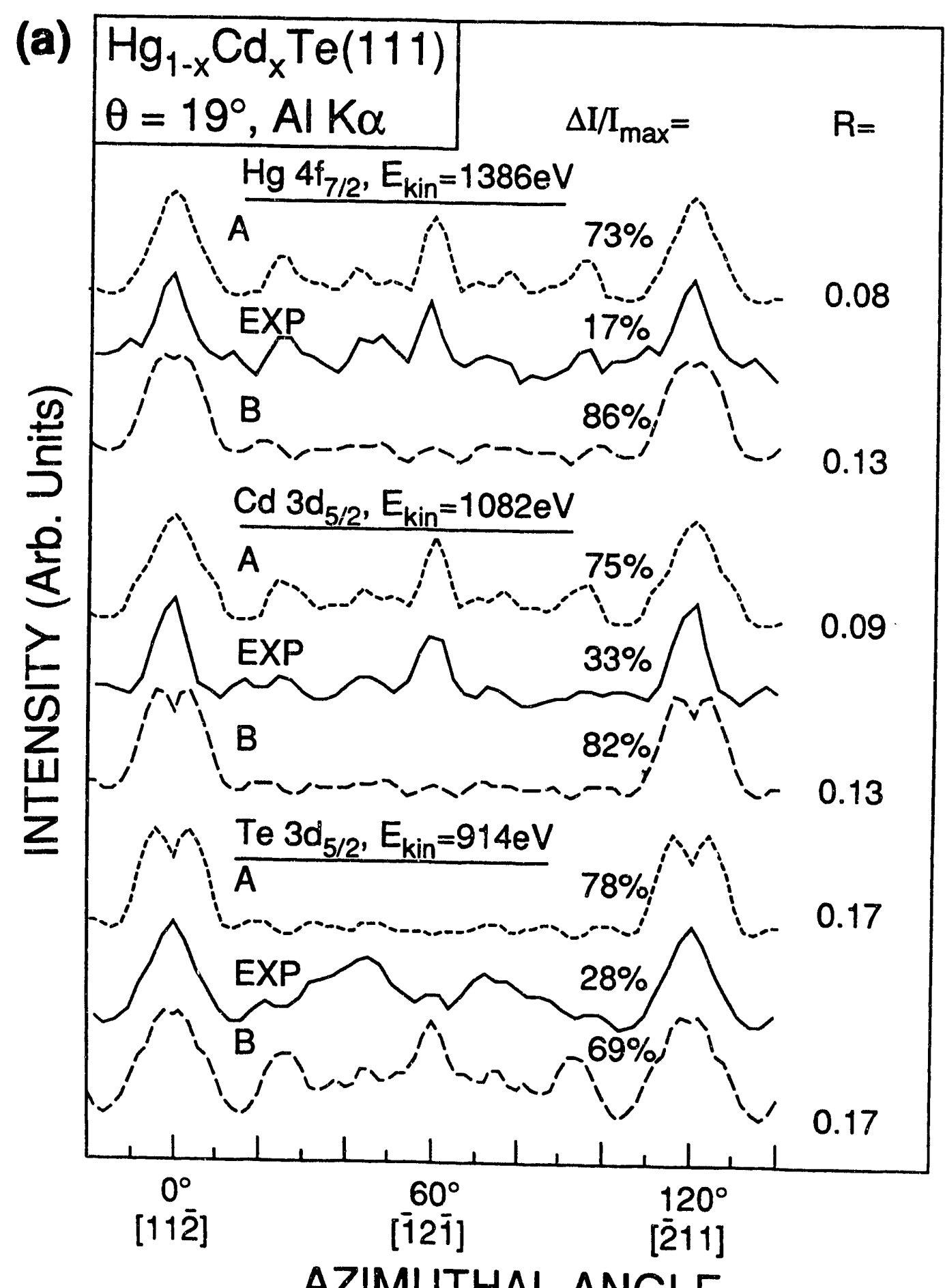

XBL $9211-2483$

Figure 5a 


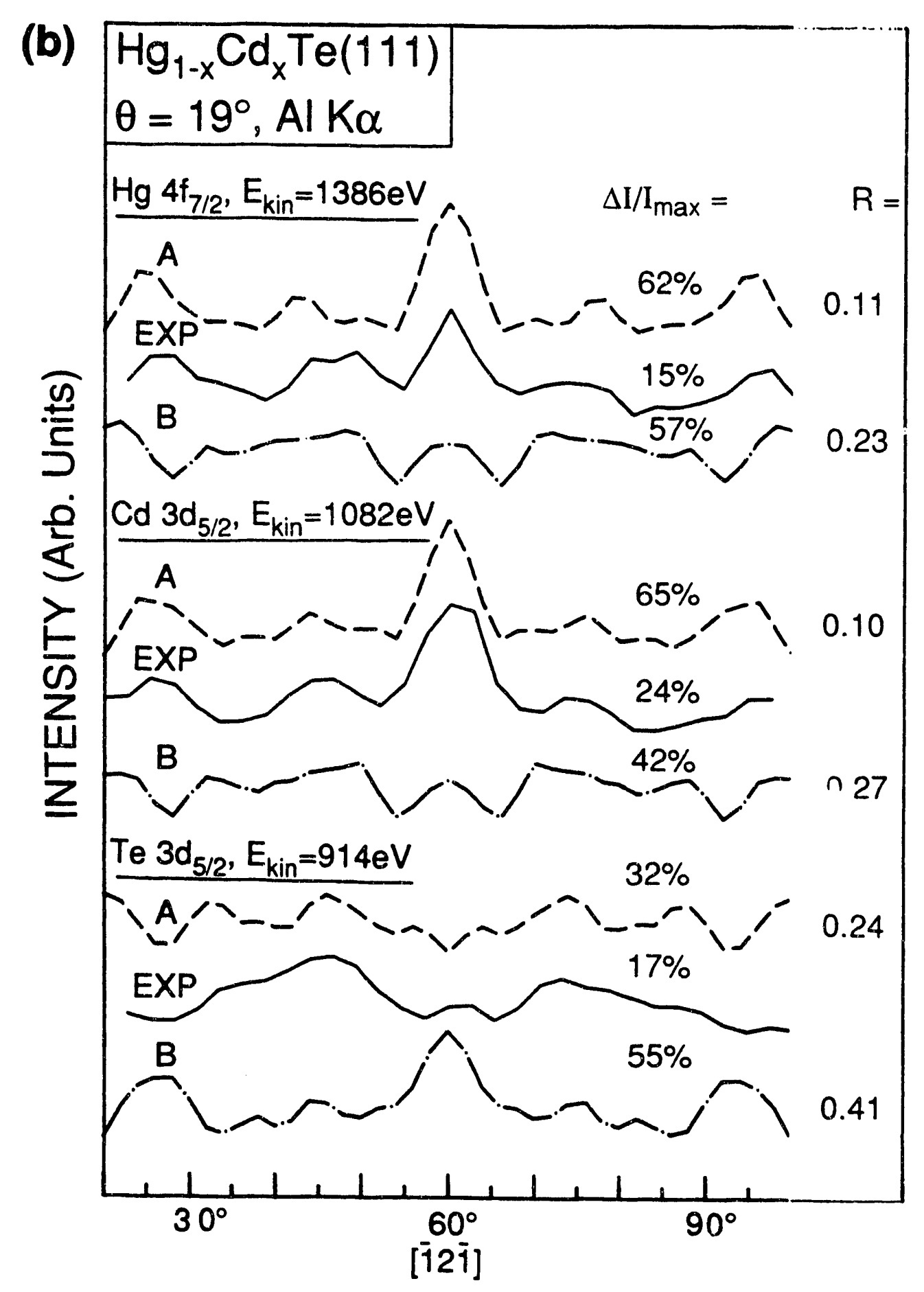

AZIMUTHAL ANGLE 


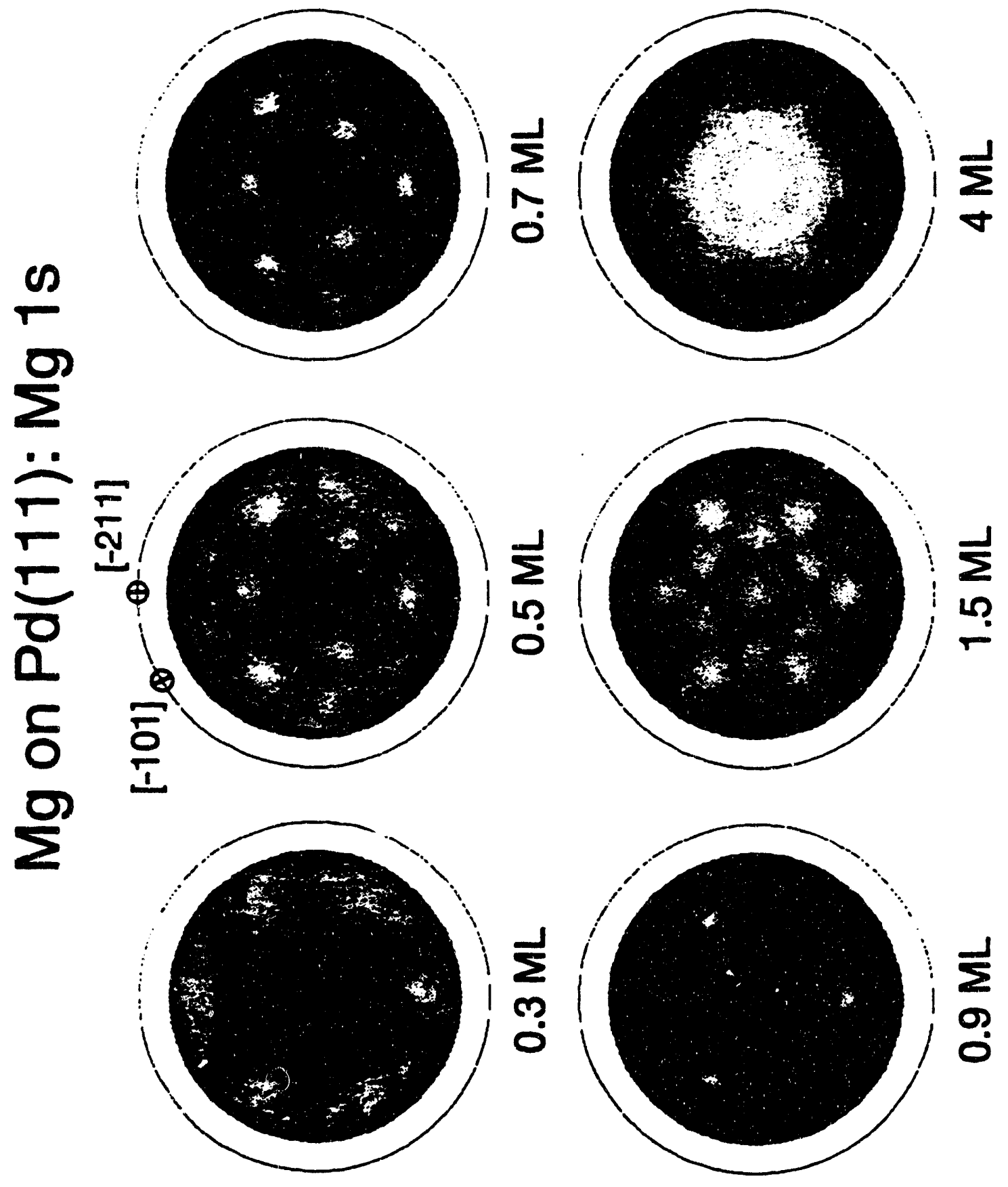

Figure 6 
$\mathrm{Cl}$ on Ni(001): $\mathrm{Cl}$ 1s emission, [111] direction, $120 \mathrm{~K}$

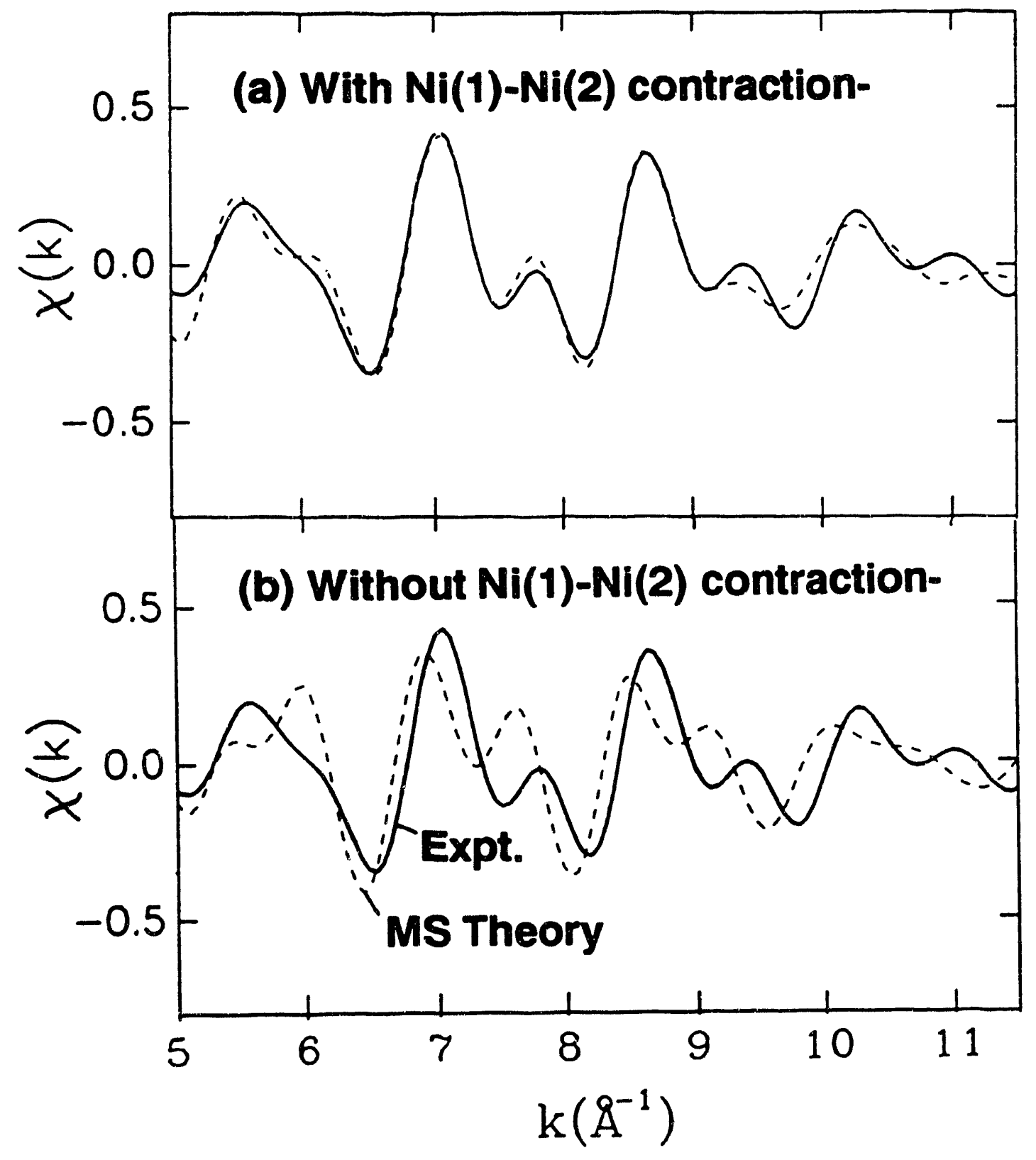

XBL $9211-2489$

Figure 7 


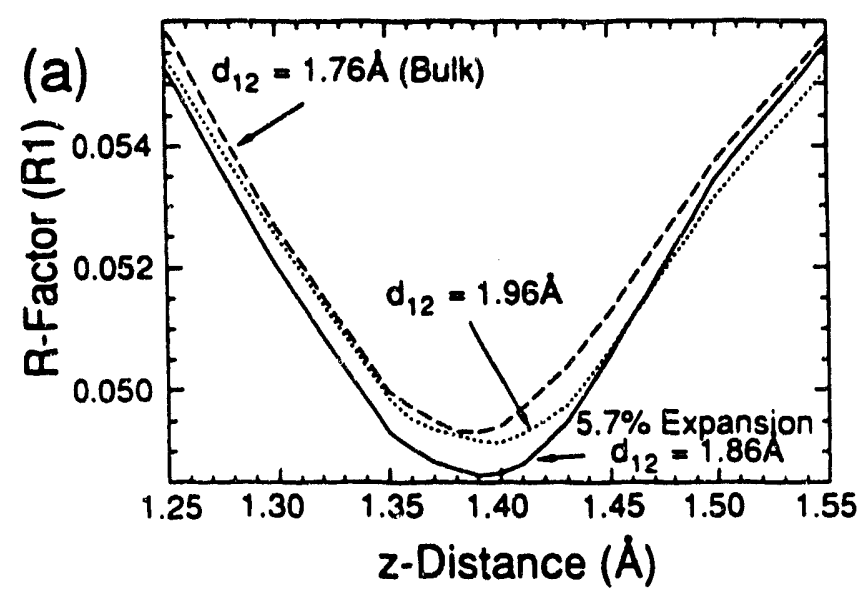

(b)

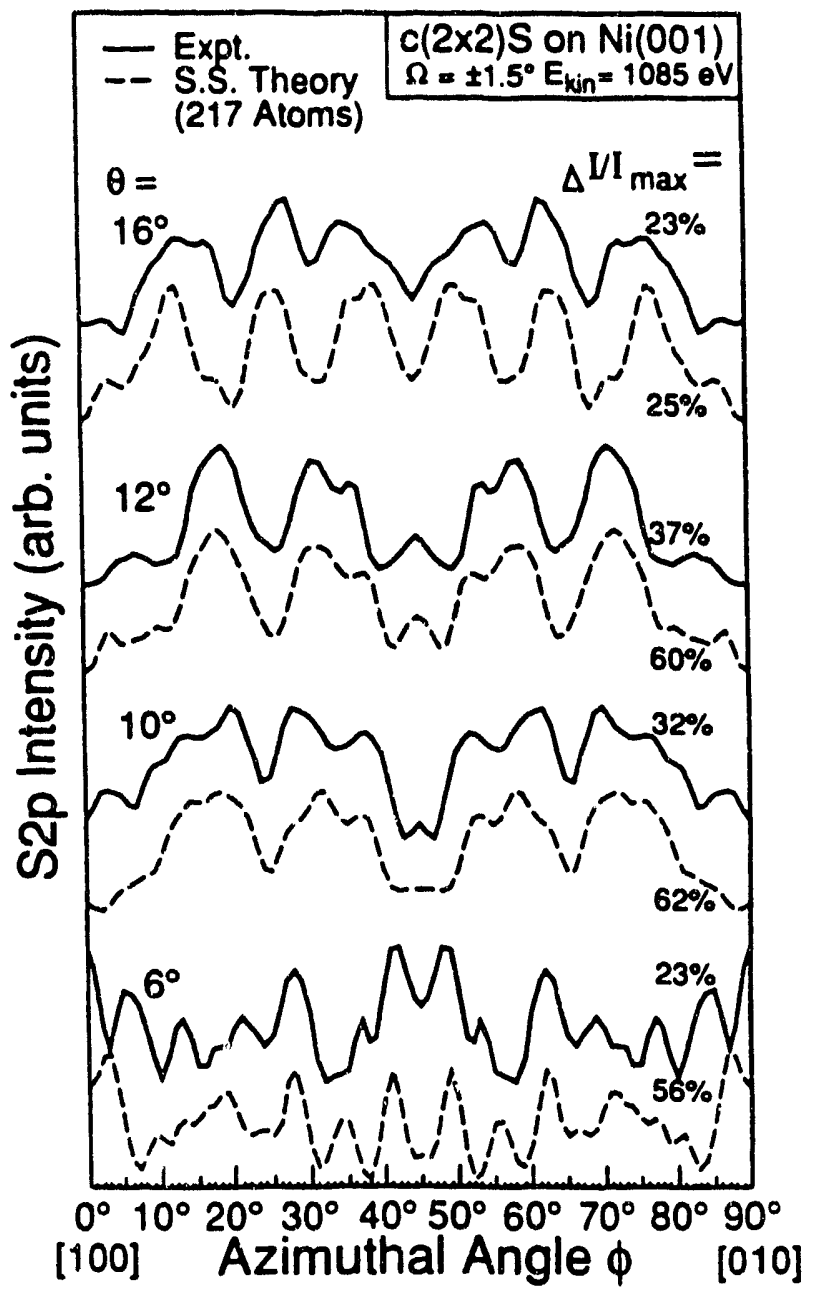

XBL 9211-2485

Figure 8 


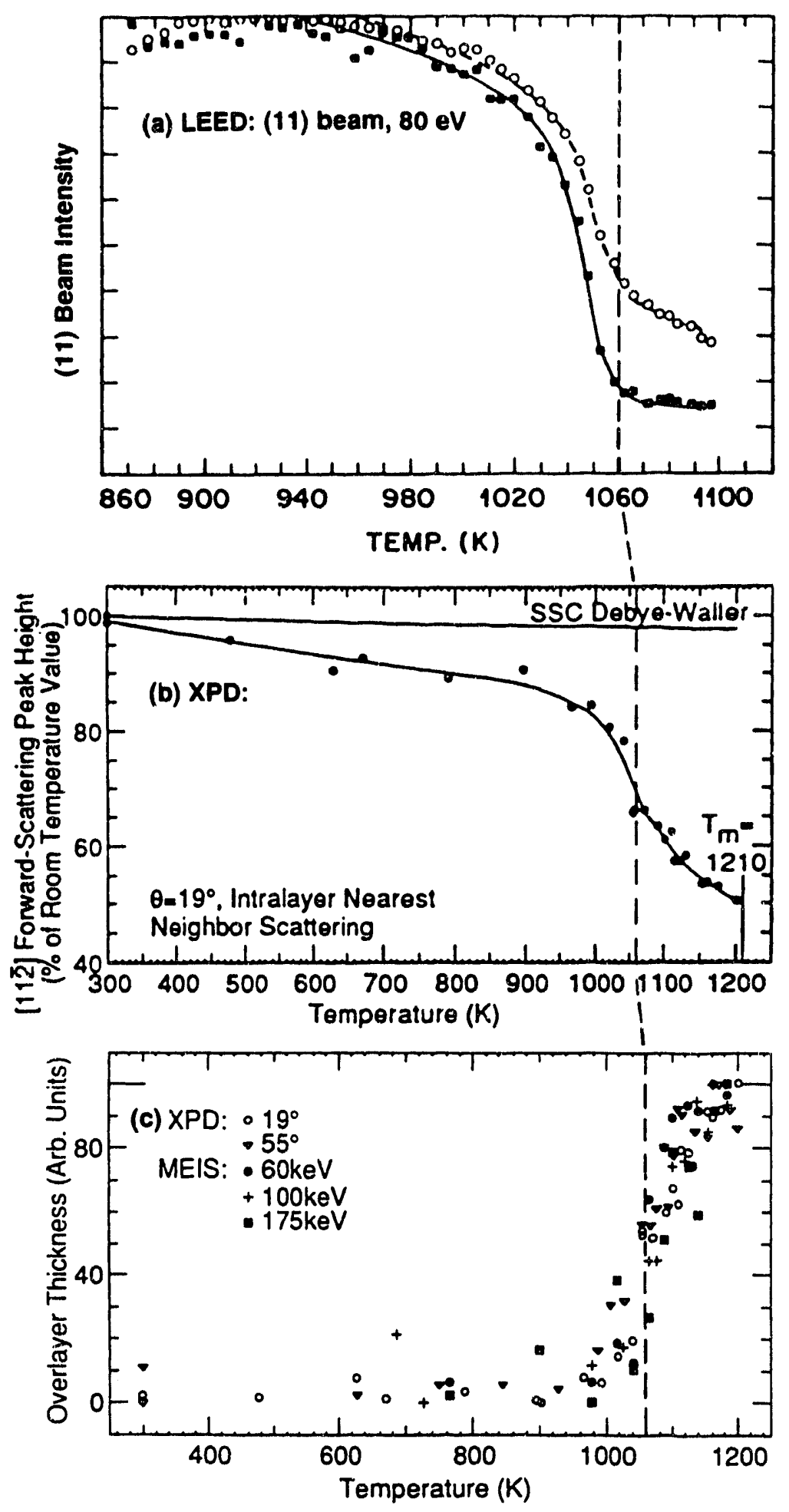

X8L 9211-2491

Figure 9 
(a) $\chi\left(k_{x}, k_{y}\right)$, Experiment, $300 \mathrm{~K}$

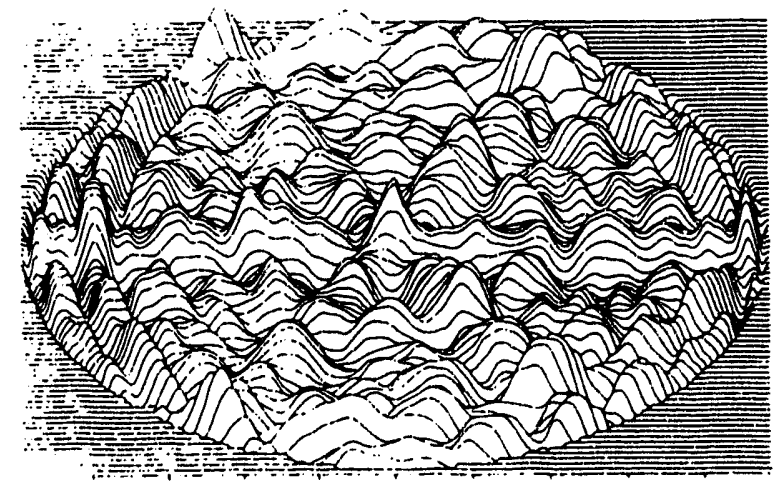

(b) $\chi\left(k_{x}, k_{y}\right)$, Experiment, $970 \mathrm{~K}$

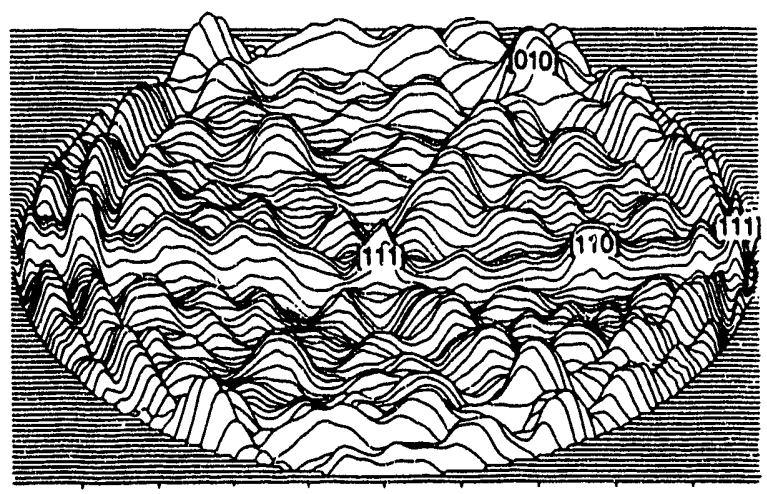

(c) $\chi\left(k_{x}, k_{y}\right)$, Experiment, $1130 \mathrm{~K}$

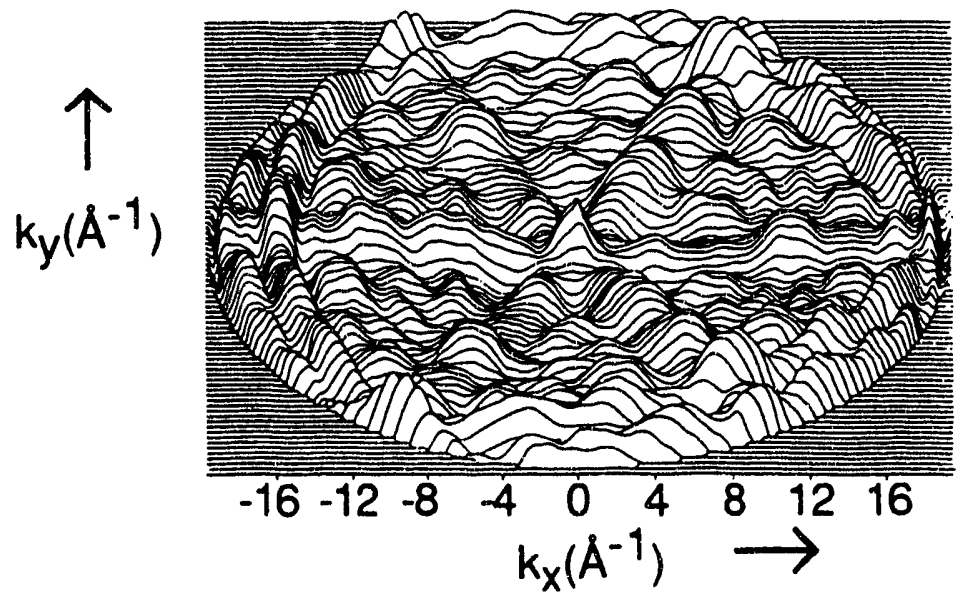

XBL 927-1674

Figure 10 

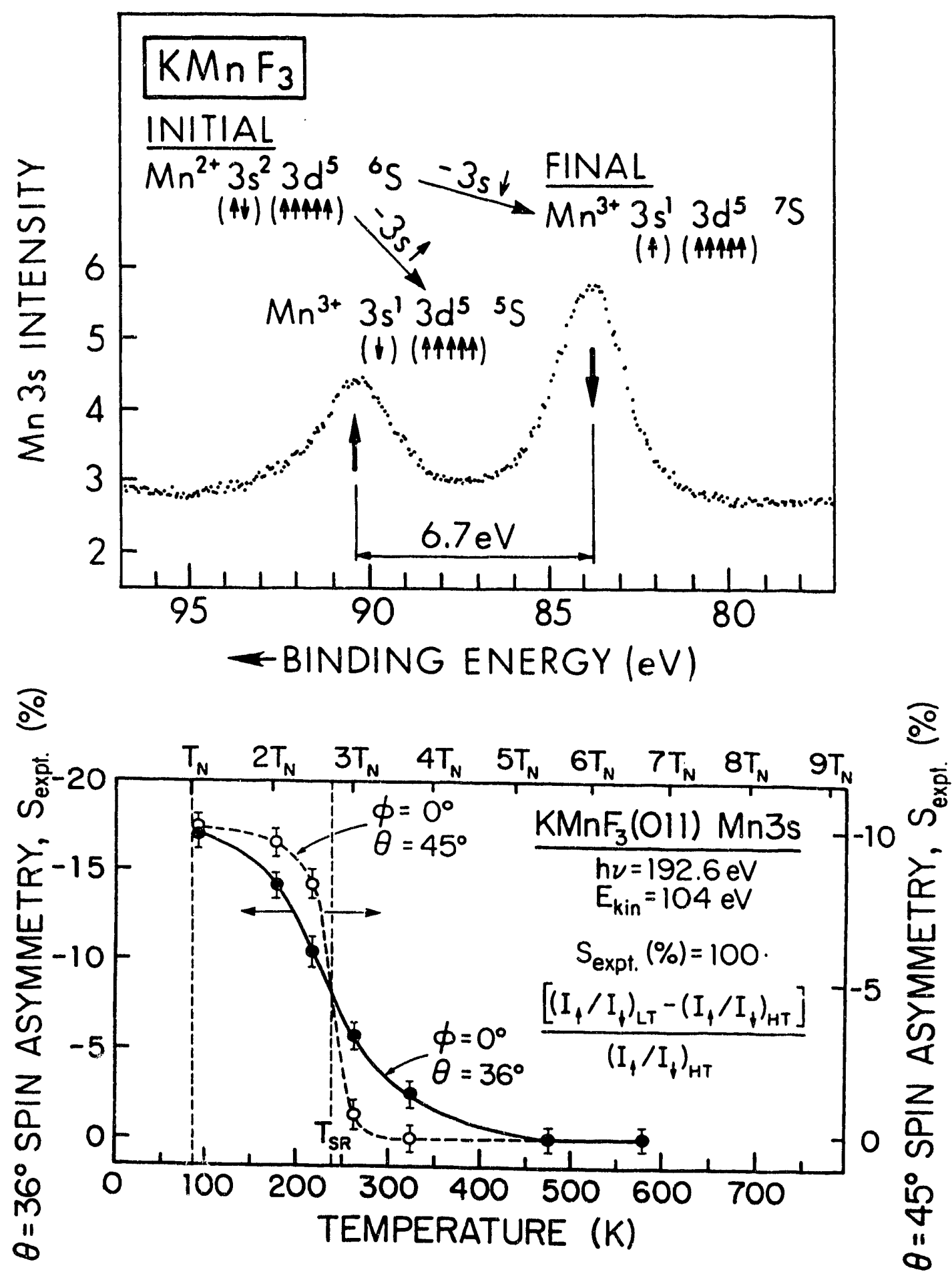


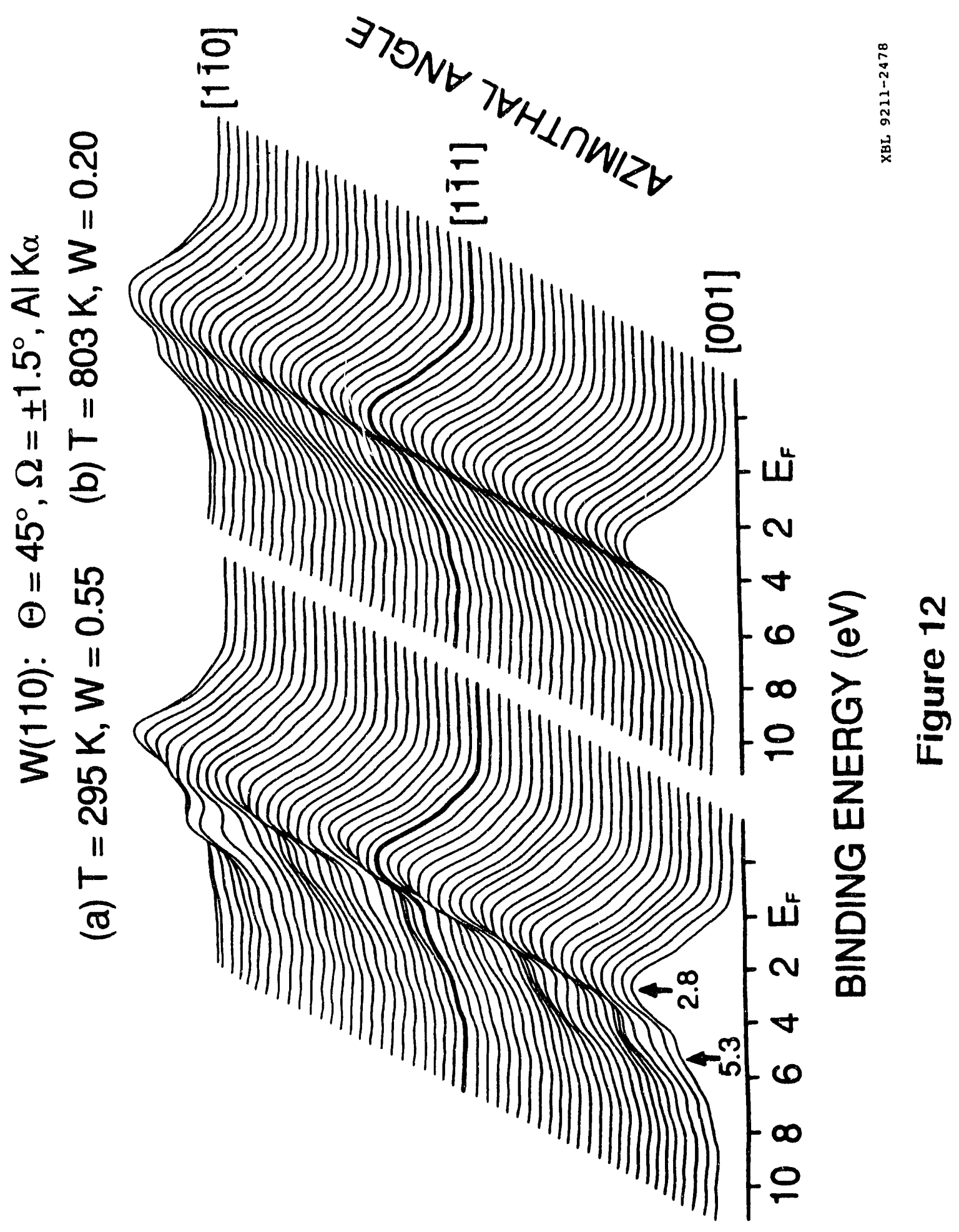




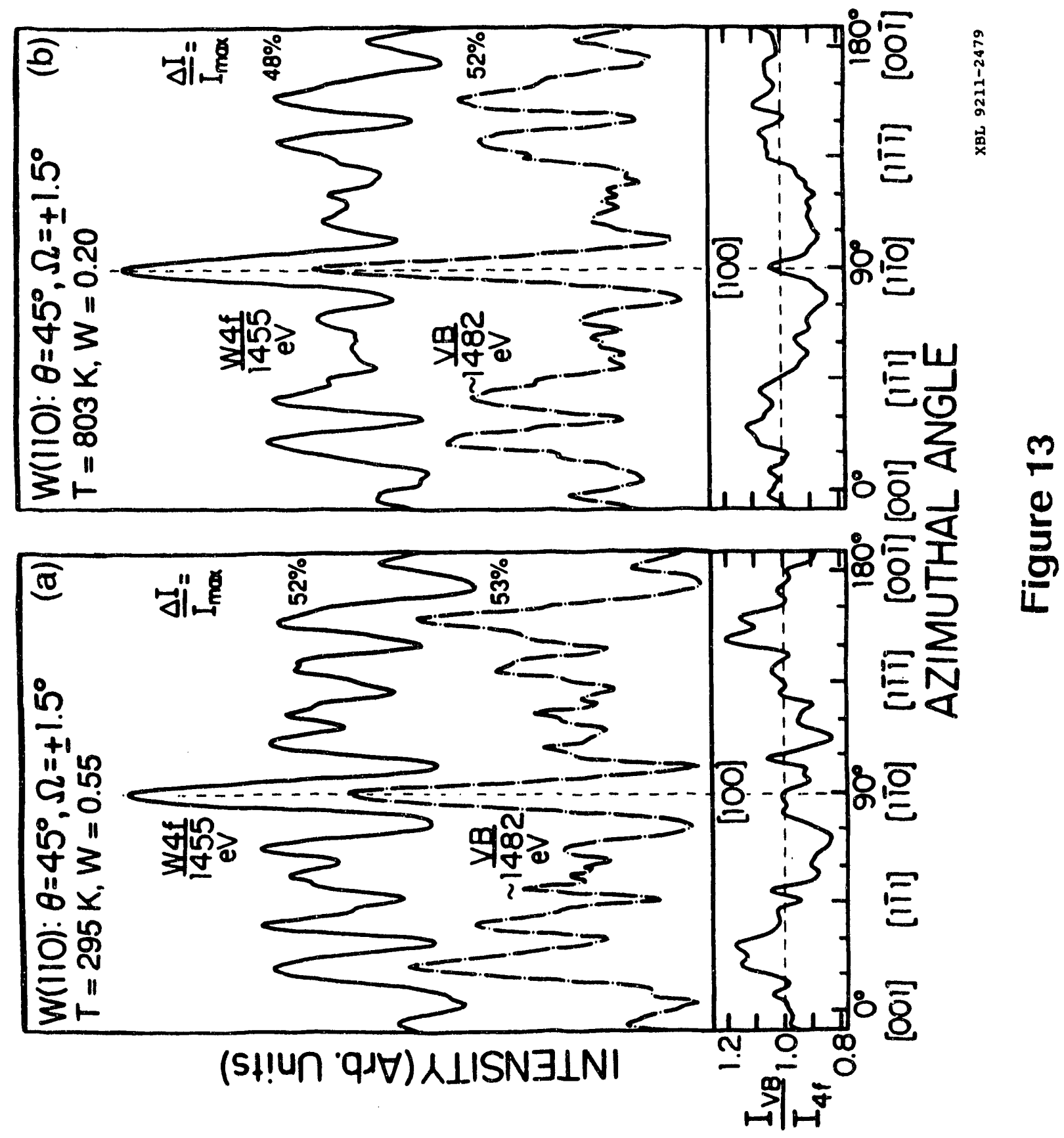



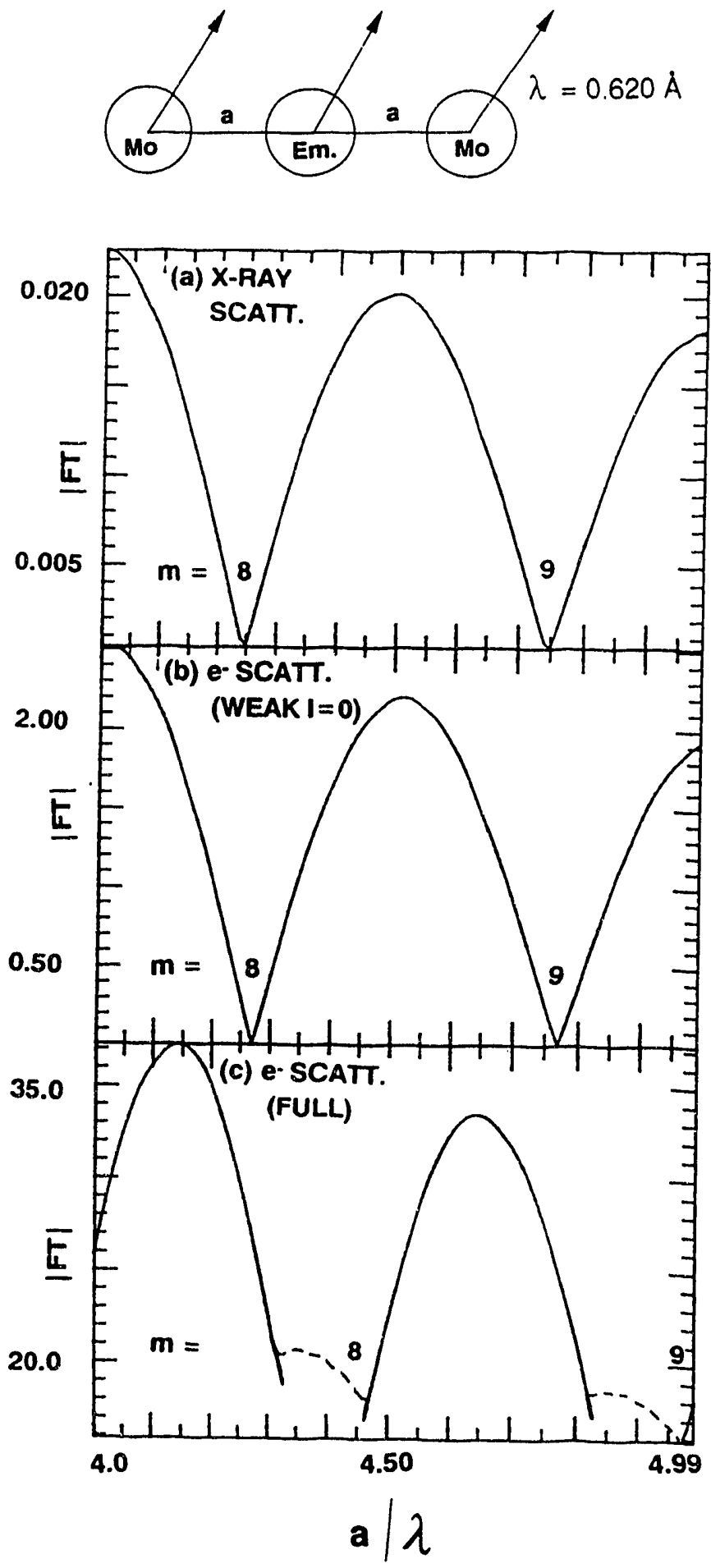

XBL $9211-2487$

Figure 14 
$-63$.

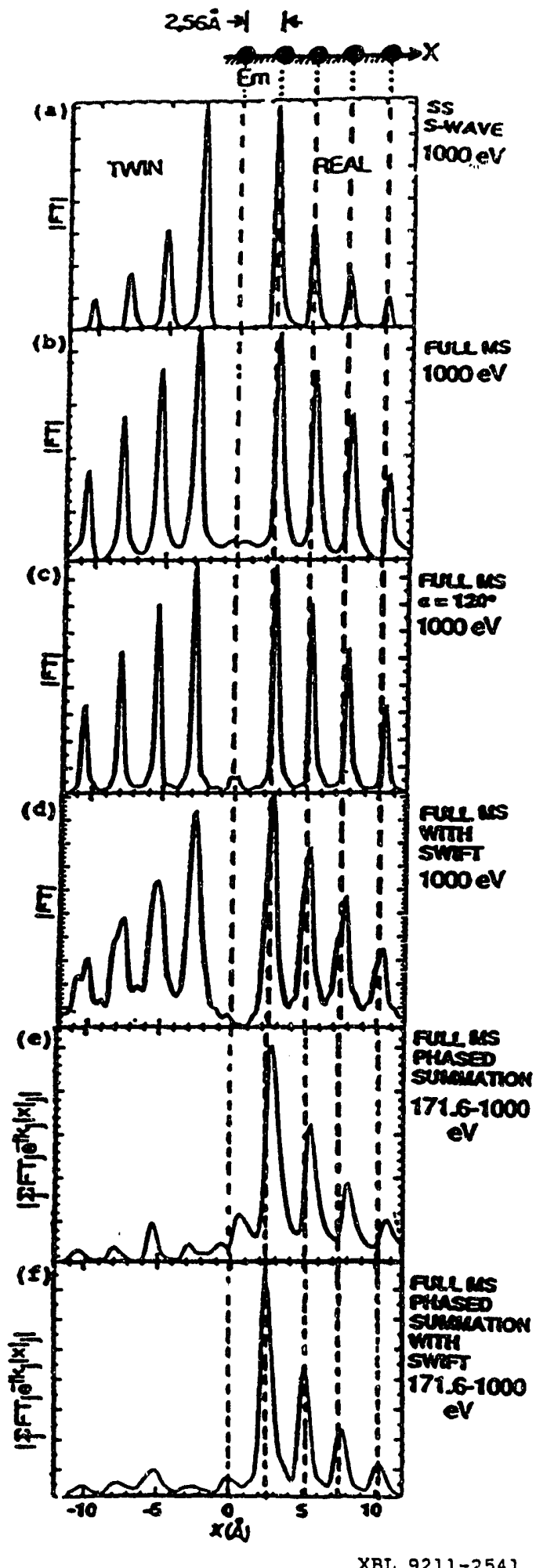

Figure 15 
(a) |FT |, XY PLANE, EXPERIMENT:

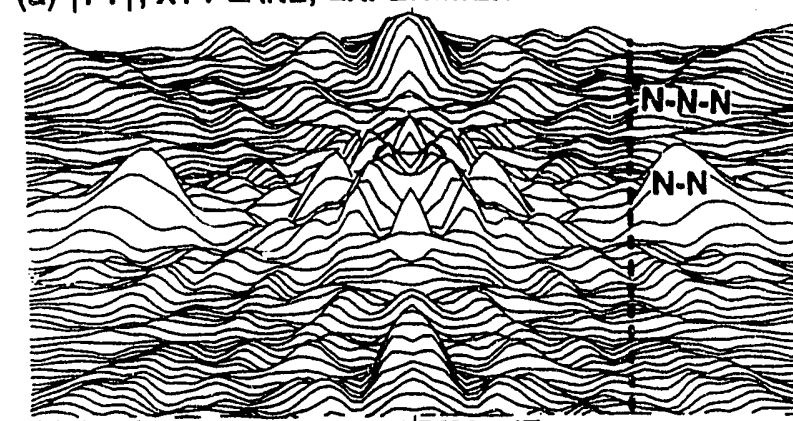

UNCORRECTED

RIGHT HALF HOLOGRAM

$E=1327 \mathrm{eV}$

(b) |FT|, XY PLANE, EXPERIMENT:

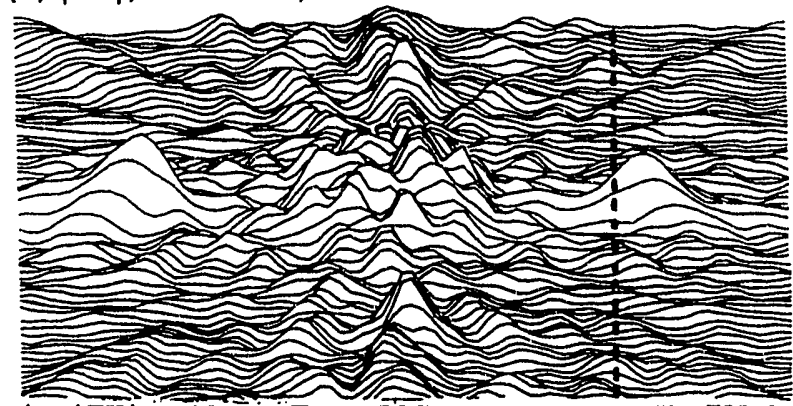

SWIFT

CORRECTED

RIGHT HALF

HOLOGRAM

$E=1327 \mathrm{eV}$

(c) |FT|, XY PLANE, THEORY:

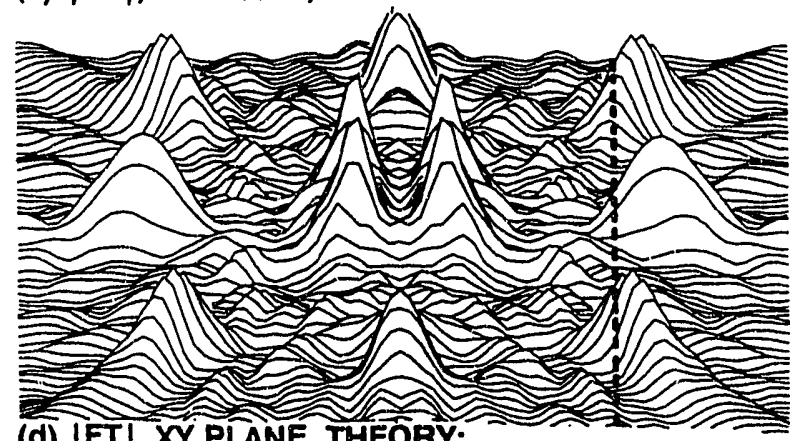

UNCORRECTED

RIGHT HALF

HOLOGRAM

$E=1327 \mathrm{eV}$

(d) |FT|, XY PLANE, THEORY:

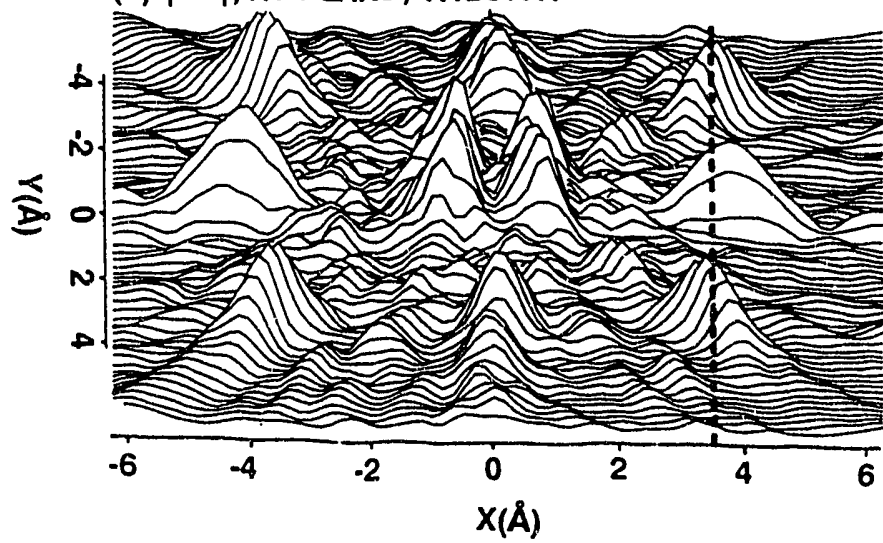

SWIFT CORRECTED RIGHT HALF HOLOGRAM $E=1327 \mathrm{eV}$

XBL $9211-2488$

Figure 16 


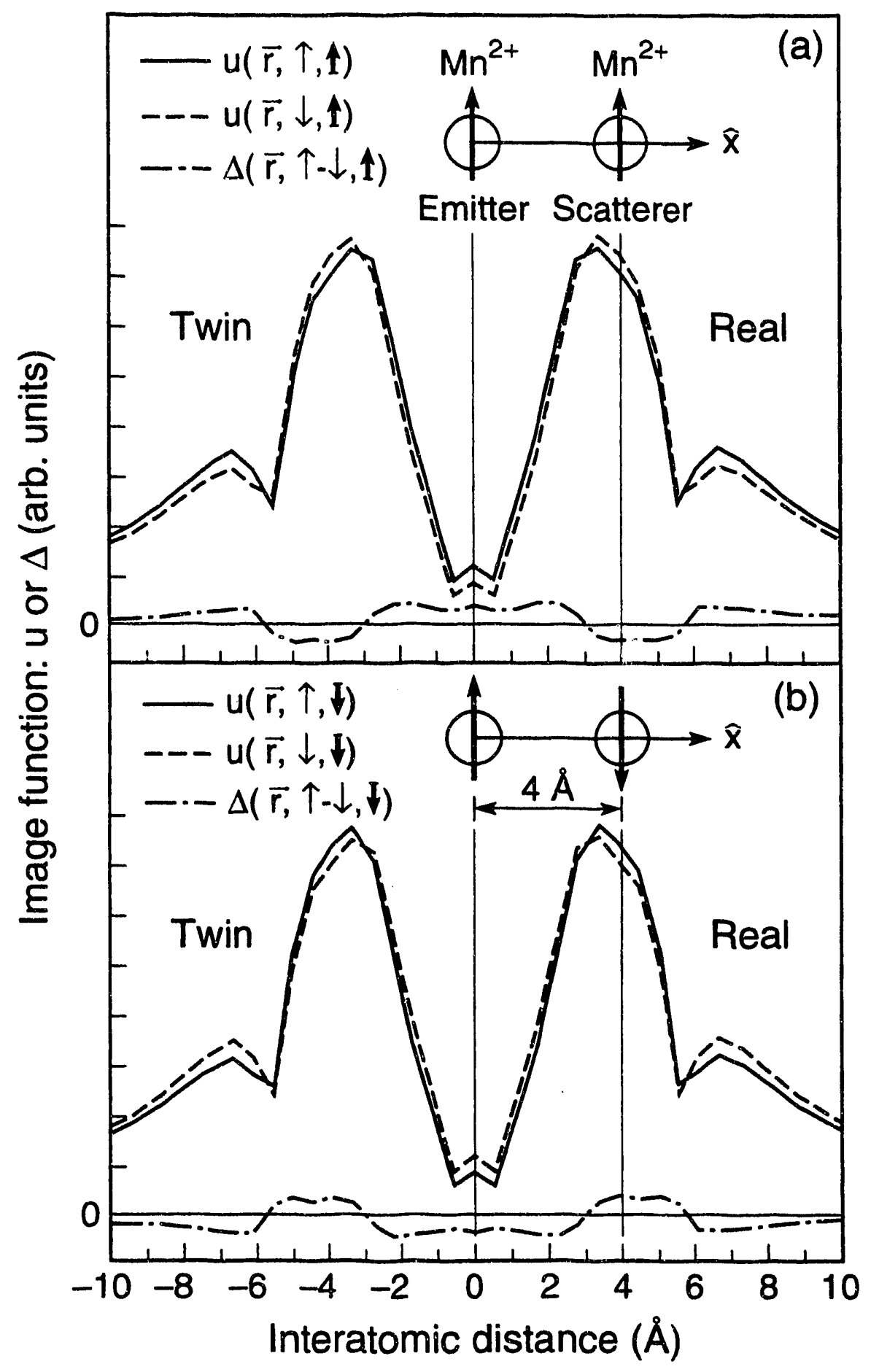

Figure 17 

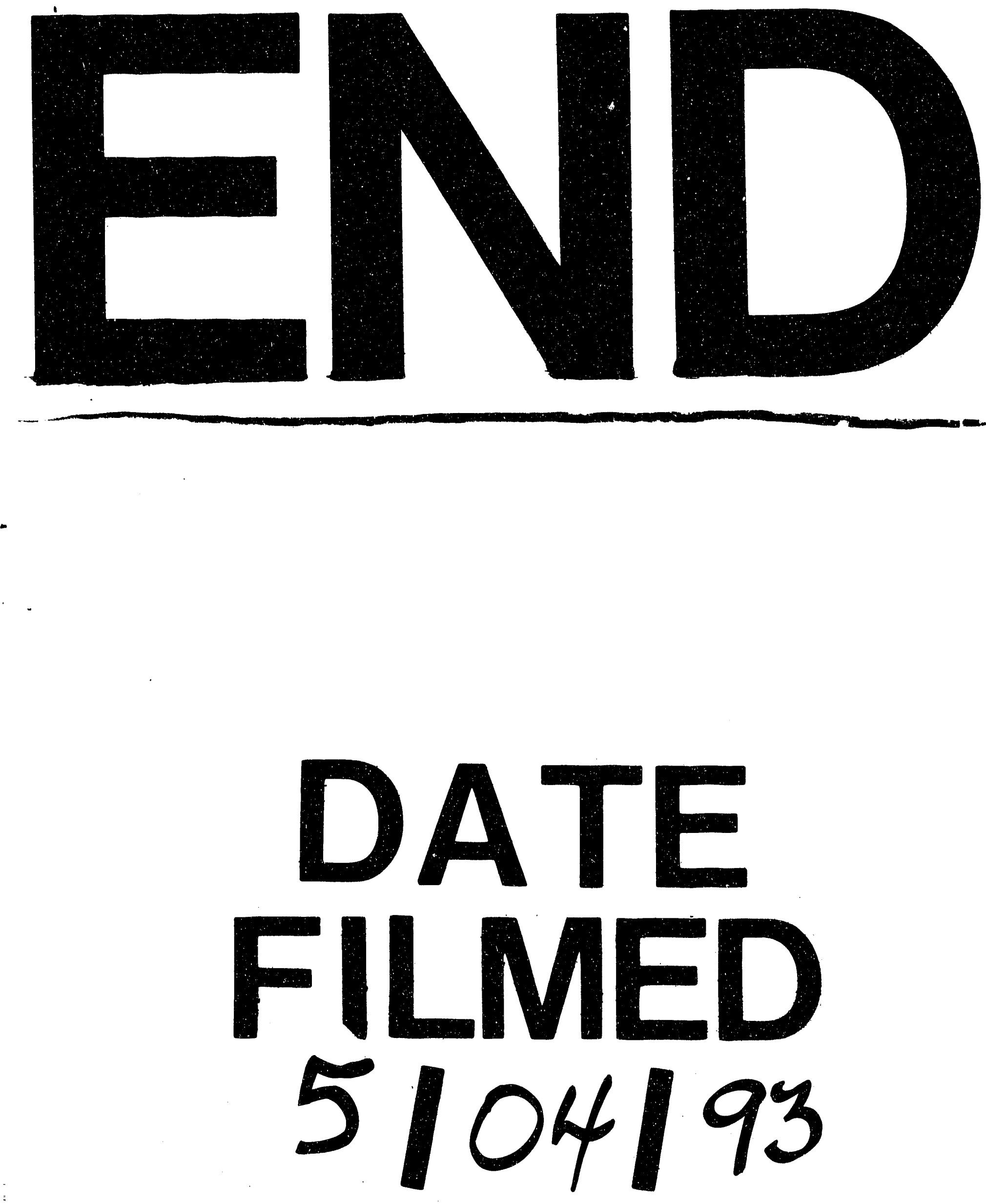
1 